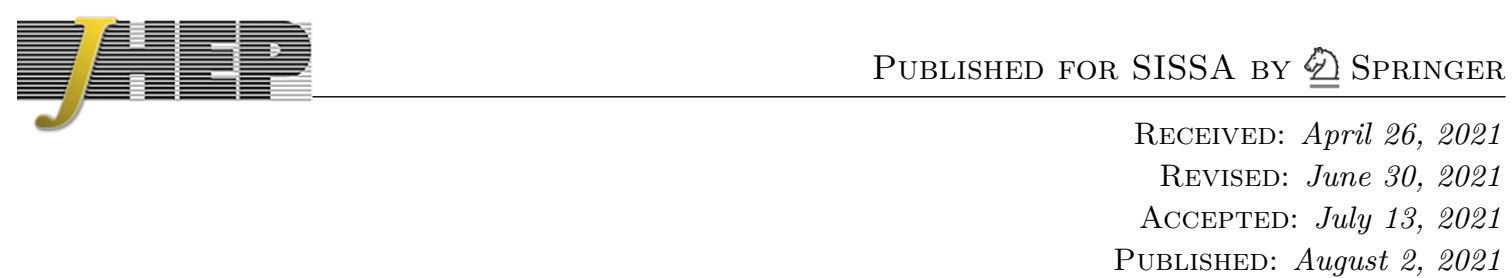

\title{
What is Leading Order for LFV in SMEFT?
}

\section{Marco Ardu and Sacha Davidson}

LUPM, CNRS, Université Montpellier,

Place E. Bataillon, F-34095 Montpellier, Cedex5, France

E-mail: marco.ardu@umontpellier.fr, s.davidson@lupm.in2p3.fr

ABstract: Upcoming searches for lepton flavour change (LFV) aim to probe New Physics (NP) scales up to $\Lambda_{N P} \sim 10^{4} \mathrm{TeV}$, implying that they will be sensitive to NP at lower scales that is suppressed by loops or small couplings. We suppose that the NP responsable for LFV is beyond the reach of the LHC and can be parametrised in Effective Field Theory, introduce a small power-counting parameter $\lambda$ (à la Cabibbo-Wolfenstein), and assess whether the existing dimension six operator basis and one-loop RGEs provide a good approximation for LFV. We find that $\mu \leftrightarrow e$ observables can be sensitive to a few dozen dimension eight operators, and to some effects of two-loop anomalous dimensions, for $\Lambda_{N P} \lesssim 20-100 \mathrm{TeV}$. We also explore the effect of some simplifying assumptions in the one-loop RGEs, such as neglecting flavour-changing effects.

KEYwords: Beyond Standard Model, Effective Field Theories

ArXiv EPrint: 2103.07212 


\section{Contents}

1 Introduction 1

2 Power-counting 3

2.1 Notation 3

2.2 The power-counting scheme 4

$\begin{array}{lll}2.3 & \text { Examples } & 7\end{array}$

3 Questions 10

3.1 Dimension eight operators 10

3.2 2-loop anomalous dimensions? 12

3.3 CKM 14

$\begin{array}{lll}3.4 & \text { LFV Yukawa couplings } & 16\end{array}$

$\begin{array}{lll}\text { 3.5 } & \text { LFV with } \tau \mathrm{s} & 18\end{array}$

$\begin{array}{lll}4 & \text { Summary } & 18\end{array}$

A Some LFV Operators of dimension eight 20

A.1 Dimension eight not present at dimension six $\quad 21$

$\begin{array}{lll}\text { A.1.1 Four-fermion } & 21\end{array}$

A.1.2 Two-lepton operators $\quad 21$

A.2 Dimension eight operators present at dimension six 22

A.2.1 Four-fermion 22

A.2.2 Two-lepton operators 22

B Tree matching at $m_{W}$ with LFV operators to dimension eight 23

B.1 Dipoles and two-photon(gluon) 23

B.2 Four-Lepton 24

B.3 Two-lepton two-quark 25

\section{Introduction}

Perturbation theory is a widely used tool in the Standard Model (SM), New Physics (NP) models and many other areas. In a given perturbative expansion, the first non-vanishing term, sometimes called the leading order contribution, is often simple to compute. However, when a calculation simultaneously involves many perturbative expansions, it can be more challenging to identify the "leading" or dominant contribution.

In this manuscript, we study perturbative expansions in the Lepton Flavour Changing (LFV) part of the Lagrangian [1, 2] of the Standard Model Effective Field Theory 
(SMEFT). We restrict to LFV operators for two reasons; firstly, they must exist because the observations of neutrino oscillations demonstrate that leptons change flavour (for a review of LFV in the $\mu \leftrightarrow e$ sector, see [3]). And secondly, LFV operators are simpler than generic operators, because SM loop effects, included via Renormalisation Group Equations (RGEs), cannot change lepton flavour, so the flavour of at least two legs of each operator remains fixed.

There are many perturbative expansions in SMEFT: the EFT expansion in the ratio of weak to New Physics scales $v^{2} / \Lambda_{N P}^{2}$, as well as the SM expansions in loops, in the $\mathcal{O}(1)$ gauge and Higgs self-couplings and in the exceptionally hierarchical Yukawa couplings, and also in mixing angles. So it is not obvious to find the leading effects. For example, it was noticed long ago by Bjorken and Weinberg [4], in the SM extended with a second Higgs $H$ with LFV couplings $Y_{\mu e} \bar{\ell}_{\mu} H P_{R} e$, that the one-loop amplitude for $\mu \rightarrow e \gamma$ is suppressed by two lepton Yukawas, so is smaller than two-loop ("Barr-Zee" [5]) contribution:

$$
\mathcal{A}_{1-\text { loop }} \propto \frac{e y_{\mu} Y_{\mu \mu}^{*} Y_{\mu e}}{16 \pi^{2} M_{H}^{2}}, \quad \mathcal{A}_{2-\text { loop }} \propto \frac{e y_{t} g^{3} Y_{\mu e}}{\left(16 \pi^{2}\right)^{2} M_{H}^{2}} .
$$

However, this leading (although two-loop) contribution was missed in part of the subsequent literature.

Various powercounting schemes have been introduced to organise perturbative calculations in flavour physics. For instance, in the quark flavour sector below the weak scale, the Wolfenstein parametrisation of the CKM matrix [6] in powers of Cabibbo's $\lambda \sim 0.22$, allows to guess the order of diagrams [7,8]. And above the weak scale, there are schemes such as Frogatt-Nielsen charges [12] and Minimal Flavour Violation [13] (see also the more general framework introduced in [14] for $B$-anomalies). Below the weak scale, a powercounting recipe for flavour is sufficient to organise a calculation, because the mass scales for the EFT are known, and the remaining couplings are few: in the RGEs for four-quark operators, QED effects can be included at appropriate subleading order in the expansion in $\alpha_{s} \log [7,9]$. For LFV below the weak scale, the "leading order" operators and RGEs have been assembled: observables can be parametrised with three and four-point functions, which correspond to operators of dimensions five to eight (see eg [10] for a list), and the "leading order" RGEs, which include two-loop vector to dipole mixing, are given in [11]. However, above the weak scale, the situation is complicated by the dynamical Higgs and $\mathrm{SU}(2)$ gauge bosons, which introduce more particle mixing in the RGEs, and also by our ignorance of the mass scale of new particles, $\Lambda_{\mathrm{NP}}$.

In this manuscript, we suppose that New Physics is "beyond the LHC", which is taken to mean $\Lambda_{N P}>4 \mathrm{TeV}$, and introduce in section 2 a generalisation of the Wolfenstein counting $^{1}$ that parametrises the expansions in all the SM parameters of SMEFT, as well as the scale ratio $v / \Lambda_{\mathrm{NP}}$, in terms of a single power-counting parameter $\lambda \sim 0.2$. For any operator, this scheme allows to identify the "leading" contribution to a given process among those that could arise at different orders in the multiple perturbative expansions. It also allows to classify the contributions of various operators to a process according to

\footnotetext{
${ }^{1}$ We thank Junji Hisano for proposing the original scheme.
} 
the order in $\lambda$, and estimate when a process can have sensitivity to an operator. So in section 3 , the power-counting scheme is used to address four questions:

1. Are dimension six operators sufficient to parametrise LFV, or can observables be sensitive to dimension eight operators?

2. Does one need two-loop anomalous dimensions in the RGEs?

3. Are LFV observables sensitive to the effects of CKM angles in the RGEs, or can the quark Yukawa matrices be approximated as diagonal?

4. If the dimension six operator $H^{\dagger} H \bar{\ell} H e$ is present, it contributes to the charged lepton mass matrix when the Higgs has a vev, so the lepton mass eigenstates are not the eigenstates of the lepton Yukawa matrix $Y_{e}$ that appears in the RGEs. How should this be accounted for?

The results are summarised in section 4 . The powercounting suggests that in the $\mu \leftrightarrow e$ sector, upcoming data could be sensitive to some dimension eight operators, and some $\mathcal{O}\left(\log /\left(16 \pi^{2}\right)^{2}\right)$ effects, for $\Lambda_{\mathrm{NP}} \lesssim 50 \rightarrow 100 \mathrm{TeV}$ (see the estimates ${ }^{2}$ in tables 5 and 6). The relevant dimension eight operators are listed in appendix A, and their (treelevel) matching onto the EFT below $m_{W}$ is given in appendix B. For $\Lambda_{\mathrm{NP}} \gtrsim 50(\rightarrow 100) \mathrm{TeV}$ in the $\mu \leftrightarrow e$ sector, and for all considered scales in the $\tau \leftrightarrow \ell$ sector $\left(\Lambda_{\mathrm{NP}} \gtrsim 4 \mathrm{TeV}\right)$, the powercounting suggests that the one-loop RGEs for dimension six operators are sufficient.

\section{Power-counting}

We want to connect low-energy LFV processes with the operator coefficients in the SMEFT. In a top-down sense, this means we want to estimate the "leading" or largest contribution of each operator coefficient to each observable, or equivalently from a bottom-up perspective, the best sensitivity of each observable to each operator.

\subsection{Notation}

We write the SM Lagrangian in notation similar to $[15,16]$; the differences are that the index order on Yukawas is doublet-singlet, and $\lambda$ will be the small power counting parameter, rather than the Higgs self-coupling.

Operators that change lepton flavour (but not number) arise at dimension $\geq 6$ in SMEFT, and are added to the Lagrangian in the basis of $[1,2]$, with coefficients written as a dimensionless $C$ divided by appropriate factors of a mass scale $\Lambda$ :

$$
\mathcal{L}_{S M E F T}=\mathcal{L}_{S M}+\left\{\frac{1}{\Lambda^{2}} \sum_{I} C_{I}^{\zeta} \mathcal{O}_{I}^{\zeta}+\frac{1}{\Lambda^{4}} \sum_{K}{ }^{(8)} C_{K}^{\zeta} \mathcal{O}_{K}^{(8) \zeta}+\text { h.c. }+\ldots\right\}
$$

where $\Lambda$ is $v=174 \mathrm{GeV}$ in the experimental constraints on coefficients $\left(1 / v^{2}=2 \sqrt{2} G_{F}\right)$, but it is sometimes convenient in the powercounting to take $\Lambda$ to be the scale $\Lambda_{\mathrm{NP}}$ of New

\footnotetext{
${ }^{2}$ Only a few $\mu \rightarrow e$ operators involving $\bar{t} t$, such as $H^{\dagger} H\left(\bar{\mu} \sigma P_{R} e\right)\left(\bar{q}_{t} \sigma P_{R} t\right)$, could contribute up to $\Lambda_{\mathrm{NP}} \lesssim$ $100 \mathrm{TeV}$.
} 
LFV Physics. So for $\Lambda=v$, the powers of $v^{2} / \Lambda_{\mathrm{NP}}^{2}$ are included the coefficients $C$. The coefficient subscripts label the gauge structure, and the superscript $\zeta$ is the flavour of the fermions composing the operator in order of appearance(sometimes the LFV indices are suppressed when they are obvious). The dimension six and eight operators are respectively labelled and normalised as in $[2]^{3}$ and [17].

The operators $\left\{\mathcal{O}_{I}\right\}$ represent LFV contact interactions among SM particles. Loop corrections to the operators generically diverge, so after renormalisation in $\overline{M S}$, the operator coefficients depend on the renormalisation scale $\mu$ and satisfy Renormalisation Group Equations (RGEs). These can be written for dimension six operators as

$$
\mu \frac{\partial}{\partial \mu} \vec{C}=\frac{1}{16 \pi^{2}} \vec{C} \widetilde{\Gamma}+\ldots
$$

where the operator coefficients are lined up in the row vector $\vec{C}$, and the matrix elements of $\widetilde{\Gamma}$ are the anomalous dimensions multiplied by SM couplings, currently known at one-loop (see $e g[15,16])$. The matrix $\widetilde{\Gamma}$ can be improved by including higher-loop contributions to the anomalous dimensions, and the equation can be extended by adding higher-dimensional operators (which changes its structure [18]). Eq. (2.2) can be solved numerically, or solved analytically as a "scale-ordered" exponential, or approximated by neglecting the running of SM couplings and exponentiating $\widetilde{\Gamma}$ :

$$
\vec{C}\left(\mu_{2}\right) \simeq \vec{C}\left(\mu_{1}\right)+\vec{C}\left(\mu_{1}\right) \frac{\widetilde{\Gamma}}{16 \pi^{2}} \ln \left(\frac{\mu_{2}}{\mu_{1}}\right)+\ldots
$$

This last approximation can be improved by including the running of some SM couplings, and selected $\mathcal{O}\left(\ln ^{2} /\left(16 \pi^{2}\right)^{2}\right)$ terms. The power-counting scheme introduced below is diagrammatic, so makes estimates in the spirit of an improved eq. (2.3), and aims to assist in determining which improvements should be included in the RGEs.

\subsection{The power-counting scheme}

The aim here is to construct a power-counting scheme allowing to organise the perturbative expansions that arise in Renormalisation Group running in the SMEFT above $m_{W}$. The input to this power-counting scheme should be the experimental sensitivities of one or several observables, and a list of operator coefficients. But since one of the expansion parameters, $v^{2} / \Lambda_{\mathrm{NP}}^{2}$, is unknown, we only bound it from above, and quantify the order of a coefficients contribution to an observable, as the scale up to which an $\mathcal{O}(1)$ coefficient could be probed.

We introduce a small parameter

$$
\lambda \simeq 0.2
$$

by analogy to the $\lambda$ parameter of the CKM matrix. The numerical value of powers $\lambda^{k}$ is given in table 1. The various dimensionless expansion parameters that occur in SMEFT can be associated to powers of $\lambda$ as discussed below (the recipe is summarised in table 2).

\footnotetext{
${ }^{3}$ The hermitian operators are here defined with a $1 / 2$, since the hermitian conjugates are included in eq. (2.1).
} 


\begin{tabular}{|c|c|c|c|c|c|c|c|c|c|}
\hline$k=$ & 1 & 3 & 4 & 5 & 6 & 7 & 8 & 10 & 12 \\
\hline$\lambda^{k}=$ & .2 & .008 & $1.6 \times 10^{-3}$ & $3.2 \times 10^{-4}$ & $6.4 \times 10^{-5}$ & $1.28 \times 10^{-5}$ & $2.56 \times 10^{-6}$ & $1.02 \times 10^{-7}$ & $4 \times 10^{-9}$ \\
\hline$\Lambda_{\mathrm{NP}}(\mathrm{TeV})$ & & & 4.3 & & 22 & & 109 & 540 & 2700 \\
\hline
\end{tabular}

Table 1. The second line gives the numerical value of $\lambda^{k}$, for $\lambda=0.2$ and $k$ from the first line. The third line gives the value of $\Lambda_{\mathrm{NP}}$, in TeV, such that $\left(v / \Lambda_{\mathrm{NP}}\right)^{2}=\lambda^{k}($ where $v=174 \mathrm{GeV})$.

1. the gauge couplings $g_{s}, g_{2}$ and $g^{\prime}$ (of respectively QCD, SU(2) and hypercharge) are counted $\sim \mathcal{O}(1)$, and sometimes retained in the estimates (because $e^{3} \sim \lambda^{2}$ ).

2. With a Lagrangian normalised as eq. (2.1) with $\Lambda=v=174 \mathrm{GeV}$, the ratio $v^{n-4} / \Lambda_{\mathrm{NP}}^{n-4}$ is absorbed into the coefficients (where $n$ is the operator dimension). In discussing dimension eight operators, we assume a New Physics scale beyond the reach of the LHC:

$$
\Lambda_{\mathrm{NP}} \gtrsim 4 \mathrm{TeV} \quad \Rightarrow \quad \frac{v^{2}}{\Lambda_{\mathrm{NP}}^{2}} \lesssim \lambda^{4}
$$

however we leave $\Lambda_{\mathrm{NP}}>v$ undetermined in estimating the relevance of two-loop or CKM effects.

3. to each loop is attributed a factor

$$
\frac{1}{16 \pi^{2}} \sim \lambda^{3}, \quad \frac{\log }{16 \pi^{2}} \sim \lambda^{2}
$$

where the loops that appear in the RGEs are accompanied by a log, so counted with one less power. (For reference, $\ln \frac{m_{W}}{m_{\mu, \tau}} \simeq 6.7,3.85$, and $\ln \frac{4 \mathrm{TeV}}{m_{W}} \simeq 3.91$.)

4. anomalous dimensions are counted as $\mathcal{O}(1)$, despite that some can be large (this may sometimes compensate for counting gauge couplings $\sim 1$ ).

5. In the lepton sector, we work in the mass eigenstate basis for charged leptons. This would be the eigenbasis of $Y_{e}$ in the SM, but can differ in the presence of nonrenormalisable operators [19]. For instance, the operator $\left[C_{e H}\right]^{i j} / \Lambda_{\mathrm{NP}}^{2} H^{\dagger} H \bar{\ell}_{i} H e_{j}$ contributes to the charged lepton mass matrix

$$
\left[m_{e}\right]^{i j}=\left[Y_{e}\right]^{i j} v-\left[C_{e H}\right]^{i j} \frac{v^{3}}{\Lambda_{\mathrm{NP}}^{2}} .
$$

However, there is a factor of 3 in the Feynman rule of $\mathcal{O}_{e H}$, such that the coupling of leptons to the SM Higgs is

$$
[\widetilde{Y}]^{i j}=\frac{1}{\sqrt{2} v}\left([m]^{i j}-2\left[C_{e H}\right]^{i j} \frac{v^{3}}{\Lambda_{N P}^{2}}\right)
$$

so in the charged lepton mass basis, flavour-changing higgs decays probe the offdiagonal coefficients of $C_{e H}$. 
The LHC measures the yukawas of the $\tau$ and the $\mu$ to be consistent with SM expectations [20-23], and constrains the $\tau \rightarrow \ell$ flavour-changing interactions of the $125-\mathrm{GeV}$ Higgs [24, 25]:

$$
\frac{v^{2}}{\Lambda_{\mathrm{NP}}^{2}} \sqrt{\left|C_{e H}^{\mu \tau}\right|^{2}+\left|C_{e H}^{\tau \mu}\right|^{2}}<1.00 \times 10^{-3}, \quad \frac{v^{2}}{\Lambda_{\mathrm{NP}}^{2}} \sqrt{\left|C_{e H}^{\mathrm{e} \tau}\right|^{2}+\left|C_{e H}^{\tau \mathrm{e}}\right|^{2}}<1.60 \times 10^{-3} .
$$

For $\mu \rightarrow e$ flavour change, the MEG bound [26] on $\mathrm{BR}(\mu \rightarrow e \gamma)$ could probe couplings as small as [10]

$$
\frac{v^{2}}{\Lambda_{\mathrm{NP}}^{2}} C_{e H}^{\mu e}, \quad \frac{v^{2}}{\Lambda_{\mathrm{NP}}^{2}} C_{e H}^{e \mu} \sim 7.5 \times 10^{-7}
$$

(larger values could be allowed if they cancel against other contributions). These bounds imply that in the charged lepton mass eigenstate basis, the off-diagonal elements of $Y_{e}$ are small (they are comparable to the LFV coefficients $C_{e H}^{i j} v^{2} / \Lambda_{\mathrm{NP}}^{2}-$ see eq. (2.5)), so the two largest eigenvalues of $Y_{e}$ can approximately be obtained from $m_{\tau}$ and $m_{\mu}$. Assuming that the magnitude of the electron Yukawa is $\leq\left. y_{e}\right|_{\max }=m_{e} / v$, one obtains that in the mass eigenstate basis,

$$
\left[Y_{e}\right]=\left[\begin{array}{ccc}
\leq 2.9 \times 10^{-6} & <10^{-6} & <10^{-3} \\
<10^{-6} & 6.0 \times 10^{-4} & <10^{-3} \\
<10^{-3} & <10^{-3} & 1.0 \times 10^{-2}
\end{array}\right] \approx\left[\begin{array}{ccc}
\lambda^{8} & \lambda^{9} & \lambda^{4} \\
\lambda^{9} & 2 \lambda^{5} & \lambda^{4} \\
\lambda^{4} & \lambda^{4} & \lambda^{3}
\end{array}\right]
$$

6. In the quark flavour sector, the mass and Yukawa matrices select eigenbases when they are diagonalised in the generation spaces of the SM fermions. Since this manuscript is focussed on LFV, operators such as $H^{\dagger} H \bar{q} H d$ or $H^{\dagger} H \bar{q} \tilde{H} u$ are not considered, and the quark masses are assumed to arise from Yukawa couplings. So the eigenvalues of $Y_{d}$ and $Y_{u}$, evaluated at $m_{W}$, are taken as:

$$
\begin{aligned}
& \left(y_{b}, y_{s}, y_{d}\right) \approx\left(1.7 \times 10^{-2}, 3.5 \times 10^{-4}, 1.7 \times 10^{-5}\right) \approx\left(\lambda^{2} / 2, \lambda^{5}, \lambda^{7}\right) \\
& \left(y_{t}, y_{c}, y_{u}\right) \approx\left(1.0,4.0 \times 10^{-3}, 6.7 \times 10^{-6}\right) \approx\left(1, \lambda^{3} / 2, \lambda^{7} / 2\right) .
\end{aligned}
$$

where $y_{f} \equiv m_{f}\left(m_{W}\right) / v$, with $m_{f}\left(m_{W}\right)$ obtained from one-loop RGEs - eg for quarks:

$$
m\left(m_{W}\right)=m(\mu)\left[\frac{\alpha_{s}\left(m_{W}\right)}{\alpha_{s}(\mu)}\right]^{4 / \beta}
$$

with $\beta=\left(33-2 N_{f}\right) / 3 \simeq 8$, and $m(\mu)$ is from the $\mathrm{PDB}$ [27] with $\mu=m_{b}, m_{c}$ for the $b, c$ and $2 \mathrm{GeV}$ otherwise. ${ }^{4}$

The CKM matrix is approximated in terms of $\lambda$ in the usual way:

$$
V_{C K M}=\left[\begin{array}{lll}
V_{u d} & V_{u s} & V_{u b} \\
V_{c d} & V_{c s} & V_{c b} \\
V_{t d} & V_{t s} & V_{t b}
\end{array}\right]=\left[\begin{array}{ccc}
0.974 & 0.224 & -0.004 \\
-0.22 & 0.99 \pm 0.02 & 0.042 \\
0.008 & -0.04 & 1.0
\end{array}\right] \simeq\left[\begin{array}{ccc}
1 & \lambda & \lambda^{3} / 2 \\
-\lambda & 1 & \lambda^{2} \\
\lambda^{3} / 2 & -\lambda^{2} & 1
\end{array}\right]
$$

\footnotetext{
${ }^{4}$ At $m_{W}$, this gives $m_{b}=3.0 \mathrm{GeV}, m_{c}=0.7 \mathrm{GeV}, m_{s}=62 \mathrm{MeV}, m_{d}=3.0 \mathrm{MeV}, m_{u}=1.2 \mathrm{MeV}$.
} 


\begin{tabular}{|l|l|l|}
\hline loop & $\frac{1}{16 \pi^{2}}$ & $\lambda^{3}$ \\
loop*log & $\frac{\log }{16 \pi^{2}}$ & $\lambda^{2}$ \\
lepton yukawas & $y_{\tau}, y_{\mu}, y_{e}$ & $\lambda^{3}, 2 \lambda^{5}, \lambda^{8}$ \\
$\ell$ flavour change & see eq. (2.9) & \\
$d$-quark yukawas & $y_{b}, y_{s}, y_{d}$ & $\lambda^{2} / 2, \lambda^{5}, \lambda^{7}$ \\
$u$-quark yukawas & $y_{t}, y_{c}, y_{u}$ & $1, \lambda^{3} / 2, \lambda^{7} / 2$ \\
$q$ flavour change & see eq. (2.11) & \\
\hline
\end{tabular}

Table 2. Power-counting scheme for the perturbative expansion of the SMEFT.

We will always work in the mass eigenstate bases of the singlet quarks, and the $u$-type components of the doublet quarks. So in the RGEs, the up Yukawa is a diagonal matrix $D_{u}$, and $Y_{d}=V_{C K M} D_{d}$. We choose the $\left\{u_{L}\right\}$ basis for quark doublets above $m_{W}$ for two reasons. First, flavour change in the RGEs is therefore suppressed by CKM and the small $d$-type Yukawas. Secondly, at dimension six in SMEFT, there is only a tensor operator for $u$ s $\left(\mathcal{O}_{\ell e q u(3)}\right)$, so this basis diagonalises the large mixing of this tensor to the dipole operator.

The CKM matrix is included also in matching at $m_{W}$, when the low-energy operators involving $d$-type quarks are expressed as SMEFT operators.

The above power-counting scheme is summarised in table 2, and should allow to estimate the contribution of any operator coefficient to any observable. The accuracy of the scheme is discussed at the end of the next subsection, by comparing to the solutions of the RGEs.

\subsection{Examples}

This section gives explicit examples of how the powercounting estimates are made, and compares them to the solutions of the RGEs.

We first consider $\mu \rightarrow e$ processes because the most restrictive experimental constraints on LFV arise in this sector, and upcoming experiments aim to improve the sensitivities by several orders of magnitude (see table 3 ; indeed, there are plans to reach a conversion ratio $\lesssim 10^{-18}$ for $\mu A \rightarrow e A[43]$ ). The Branching Ratios can be expressed (see $e g[3,10,42]$ ) in terms of the coefficients, evaluated at the experimental scale, of operators which contribute at tree level. For instance, the low-energy operators

$$
\delta \mathcal{L}=2 \sqrt{2} G_{F}\left(C_{D, L} m_{\mu} \bar{e} \sigma \cdot F P_{L} \mu+C_{D, R} m_{\mu} \bar{e} \sigma \cdot F P_{R} \mu\right)
$$

contribute to $\mu \rightarrow e \gamma[26]$ as

$$
B R(\mu \rightarrow e \gamma)=384 \pi^{2}\left(\left|C_{D, R}\right|^{2}+\left|C_{D, L}\right|^{2}\right)<4.2 \times 10^{-13}
$$

which gives the experimental bounds, translated into our power counting parameter $(\Lambda \sim v$ in eq. (2.1))

$$
\left|C_{D, R}\right|,\left|C_{D, L}\right|<1.05 \times 10^{-8} \sim \lambda^{11}
$$




\begin{tabular}{|l|l|l|}
\hline process & bound on BR & sensitivity to $C$ \\
\hline$\mu \rightarrow e \gamma$ & $<4.2 \times 10^{-13}[26] \rightarrow 6 \times 10^{-14}[28]$ & $C_{D} \sim \lambda^{11} \rightarrow \lambda^{12}$ \\
$\mu \rightarrow e \bar{e} e$ & $<1.0 \times 10^{-12}[29] \rightarrow 10^{-16}[30]$ & $C_{S} \sim \lambda^{8} \rightarrow \lambda^{11}$ \\
& & $C_{V} \sim \lambda^{8.5} \rightarrow \lambda^{11.5}$ \\
$\mu A \rightarrow e A$ & $<7 \times 10^{-13}[31-33] \rightarrow 10^{-16}[34-36]$ & $C_{V, D} \sim \lambda^{9.5} \rightarrow \lambda^{12}$ \\
& & $C_{S} \sim \lambda^{10.5} \rightarrow \lambda^{14}$ \\
\hline$\overline{K_{L}^{0}} \rightarrow \mu \bar{e}$ & $<4.7 \times 10^{-12}$ & $C_{P} \sim \lambda^{11.5}$ \\
& & $C_{A} \sim \lambda^{9.5}$ \\
\hline$B_{d}^{0} \rightarrow \mu^{ \pm} e^{\mp}$ & $<1 \times 10^{-9}$ & $C_{P} \sim \lambda^{7.5}$ \\
$B_{d}^{+} \rightarrow \pi^{+} \bar{\mu} e$ & $<1.7 \times 10^{-7}$ & $C_{V} \sim \lambda^{7}$ \\
\hline$B_{s}^{0} \rightarrow \mu^{ \pm} e^{\mp}$ & $<5.4 \times 10^{-9}$ & $C_{P} \sim \lambda^{7.5}$ \\
$B^{+} \rightarrow K^{+} \bar{\mu} e$ & $<9.1 \times 10^{-8}$ & $C_{V} \sim \lambda^{6.5}$ \\
\hline$D^{0} \rightarrow \mu^{ \pm} e^{\mp}$ & $<1.3 \times 10^{-8}$ & $C_{P} \sim \lambda^{6}$ \\
$D^{+} \rightarrow \pi^{+} \bar{\mu} e$ & $<1.7 \times 10^{-7}$ & $C_{V} \sim \lambda^{4}$ \\
\hline \hline$\tau \rightarrow \ell \gamma$ & $<3.3 \times 10^{-8}[37]$ & $C_{D} \sim \lambda^{7.5}$ \\
$\tau \rightarrow \ell \bar{\ell} \ell$ & $\lesssim 2 \times 10^{-8}[38] \rightarrow \lesssim 10^{-9}[39]$ & $C_{V} \sim \lambda^{5} \rightarrow \lambda^{5.5}$ \\
& & $C_{S} \sim \lambda^{4.5} \rightarrow \lambda^{5}$ \\
\hline$\tau \rightarrow \ell \pi^{0}$ & $<8.0 \times 10^{-8}[40]$ & $C_{S} \sim \lambda^{4.5}$ \\
$\tau \rightarrow \ell \eta$ & $<6.5 \times 10^{-8}[40]$ & $C_{S} \sim \lambda^{4.5}$ \\
$\tau \rightarrow \ell \rho$ & $<1.2 \times 10^{-8}[41]$ & $C_{V} \sim \lambda^{4.5}$ \\
\hline$B_{d}^{0} \rightarrow e \tau$ & $<2.8 \times 10^{-5}[27]$ & $C_{P} \sim \lambda^{5}$ \\
& & $C_{A} \sim \lambda^{4.5}$ \\
\hline
\end{tabular}

Table 3. Some current and upcoming experimental bounds on LFV Branching Ratios $(\tau \leftrightarrow \mu$ results are similar to $\tau \leftrightarrow e$ ). The third column gives the order of magnitude of dimension six operator coefficients that reproduce the experimental numbers, in powers of $\lambda \simeq 1 / 5$. The listed coefficients $C_{L o r}$ contribute to the process at tree level, are labelled by the operator's Lorentz structure, and are normalised to a scale $\Lambda=v=174 \mathrm{GeV}$ in eq. (2.1). The meson decay bounds are from [27], the coefficient sensitivities from [10, 42, 44].

The dipole is a special case, because the operators contain not only fields, but also a builtin parametric suppression factor $m_{\mu}$. This is the usual operator definition, and makes sense because in SMEFT the operator has a Higgs leg which frequently attaches to the muon line. However, in some loop diagrams (for instance Barr-Zee) the Higgs is attached to a heavier particle in a loop, so such diagrams would gain a factor $1 /\left(2 \lambda^{5}\right)$ in our powercounting scheme. For a different normalisation of the dipole operator, the power-counting sensitivity would change. For instance,

$$
\delta \mathcal{L}=2 \sqrt{2} G_{F}\left(C_{D, L} v \bar{e} \sigma \cdot F P_{L} \mu+C_{D, R} v \bar{e} \sigma \cdot F P_{R} \mu\right)
$$

gives $\left|C_{D, R}\right|,\left|C_{D, L}\right| \lesssim \lambda^{16}$. 

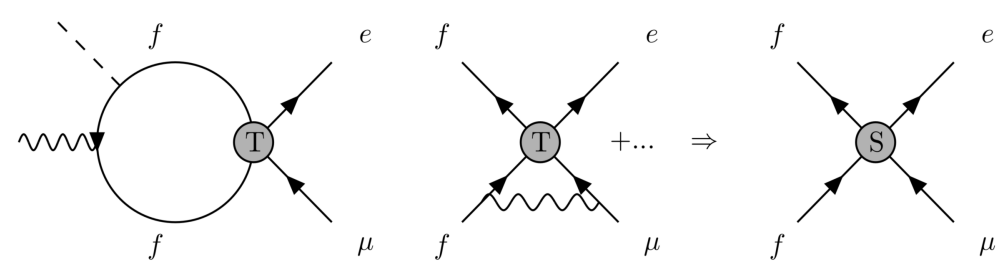

Figure 1. On the left, a diagram mixing the tensor operator to the dipole (the Higgs leg is replaced by a mass insertion in the EFT below $m_{W}$ ). On the right, one of the diagrams mixing tensor operators to scalars (the gauge boson can attach to any two legs not belonging to the same bilinear).
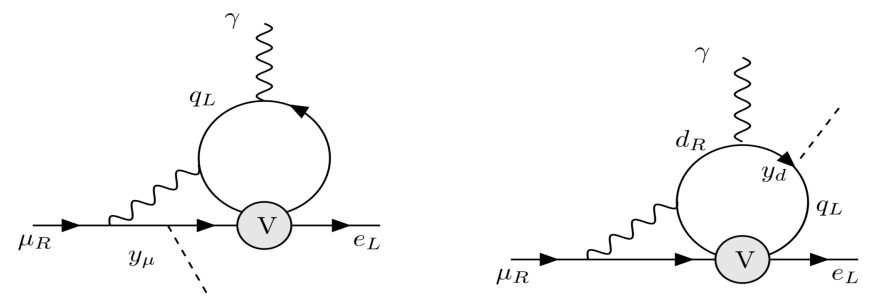

Figure 2. Representative diagrams allowing two-loop mixing of vector operators to the dipole.

\begin{tabular}{|ccc|}
\hline$f$ & Power Counting & Running \\
\hline$e$ & $\sim 20 \mathrm{TeV}$ & $\sim 13 \mathrm{TeV}$ \\
$\mu$ & $\sim 300 \mathrm{TeV}$ & $\sim 190 \mathrm{TeV}$ \\
$\tau$ & $\sim 10^{3} \mathrm{TeV}$ & $\sim 1.1 \times 10^{3} \mathrm{TeV}$ \\
$u$ & $\sim 50 \mathrm{TeV}$ & $\sim 71 \mathrm{TeV}$ \\
$d$ & $\sim 50 \mathrm{TeV}$ & $\sim 73 \mathrm{TeV}$ \\
$s$ & $\sim 200 \mathrm{TeV}$ & $\sim 330 \mathrm{TeV}$ \\
$c$ & $\sim 10^{3} \mathrm{TeV}$ & $\sim 1.7 \times 10^{3} \mathrm{TeV}$ \\
$b$ & $\sim 2 \times 10^{3} \mathrm{TeV}$ & $\sim 2 \times 10^{3} \mathrm{TeV}$ \\
\hline
\end{tabular}

Table 4. Powercounting estimates of the mixing from tensor to dipole operators below $m_{W}$, compared to the solutions of the RGEs [10, 11].

The sensitivity of $\mu \rightarrow e \gamma$ to other operators can be estimated in our power-counting scheme by drawing diagrams. For instance, tensor operators mix to the dipole via the left diagram of figure 1. Below the electroweak scale and normalizing as in eq. (2.12), the contribution to the dipole coefficient is of order

$$
\Delta C_{D} \frac{m_{\mu}}{v^{2}} \sim e \frac{\log }{16 \pi^{2}} C_{T}^{f f} \frac{m_{f}}{\Lambda_{\mathrm{NP}}^{2}} \Rightarrow \Delta C_{D} \sim e \lambda^{2} C_{T}^{f f} \frac{y_{f}}{y_{\mu}} \frac{v^{2}}{\Lambda_{\mathrm{NP}}^{2}}
$$

where $f=u, d, s, c, b, e, \mu, \tau$, and the estimate in our power-counting scheme can be obtained using table 2 . 
Scalar and vector operators can contribute to the dipole via two-loop diagrams, that arise either as one-loop mixing into the tensor, or direct mixing to the dipole at two-loop. Below the weak scale, the scalar to tensor mixing is via diagrams like the right figure 1 , where the gauge boson is a photon, which gives

$$
\Delta C_{D} \sim e^{3} \frac{\log ^{2}}{\left(16 \pi^{2}\right)^{2}} C_{S}^{f f} \frac{y_{f}}{y_{\mu}} \frac{v^{2}}{\Lambda_{\mathrm{NP}}^{2}}
$$

where now $f=u, d, s, c, b, \tau$. The vector to dipole mixing is via diagrams such as figure 2 . We estimate the diagrams on the left and right as

$$
\Delta C_{D} \sim e^{3} \frac{\log }{\left(16 \pi^{2}\right)^{2}} C_{V}\left(\frac{v}{\Lambda_{\mathrm{NP}}}\right)^{2} \times\left\{\begin{array}{c}
1 \\
\frac{y_{d}}{y_{\mu}}
\end{array}\right.
$$

so there is sensitivity to vector coefficients for scales below $10 \mathrm{TeV}$ (which is consistent with the bound in $[10,11])$.

Approximating physical predictions in terms of powers of some parameter is always somewhat arbitrary and erroneous (Indeed, although we count in $\lambda$, we allow for $\sqrt{\lambda}$ in table 3$)$. In order to test our recipe, in table 4 we compare our power-counting estimates to the solutions of the RGEs; this estimate is obtained in the EFT below $m_{W}$, for which the solution of the "leading order" RGEs is given in $[10,11]$. The table shows that our estimate of the scale $\Lambda_{\mathrm{NP}}$ where $C_{T}^{f f}$ would be $\sim 1$, (obtained by combining eq. (2.16) with column three of table 3 ), differs by at most $\sqrt{3}$ from the solution of the RGEs (this corresponds to a factor $\leq 3 \sim 1 / \sqrt{\lambda}$ for $C$, so less than an order of magnitude in the rate). For the second-order/two-loop mixing of eqns (2.17), (2.18) we find that the powercounting can mis-estimate $\Lambda_{\mathrm{NP}}$ by a factor $2-3$.

\section{Questions}

This section uses the power-counting proposal of the previous section to study what physics should be included at "leading order", in the SMEFT RGEs for LFV operators. In the first sections, the focus is on $\mu \leftrightarrow e$ flavour change, due to the sensitivity of current and upcoming experiments; the importance of dimension eight operators and two-loop anomalous dimensions for $\tau$-LFV is briefly discussed in section 3.5.

\subsection{Dimension eight operators}

This section explores when which dimension eight operators are required, and whether their RGEs are required.

We suppose that the New Physics responsable for LFV is beyond the reach of the LHC, so $\Lambda_{\mathrm{NP}} \gtrsim 4 \mathrm{TeV}$. In the normalisation convention of table 3 , this implies that coefficients of dimension eight operators are suppressed by $\sim \lambda^{8}$ :

$$
\frac{f\left(g_{N P}, \ldots\right)}{(4 \mathrm{TeV})^{4}} \mathcal{O}^{(8)}=\frac{{ }^{(8)} C}{v^{4}} \mathcal{O}^{(8)} \Rightarrow{ }^{(8)} C \lesssim \frac{v^{4}}{(4 \mathrm{TeV})^{4}} \simeq \lambda^{8}
$$


Comparing to the tree-level sensitivities given in table 3, one sees that kaon and muon decays are generically sensitive to dimension eight operators induced by new particles in the interesting mass range just beyond the reach of the LHC. Pushing the New Physics scale above $20 \mathrm{TeV}$ would give ${ }^{(8)} C \lesssim \lambda^{12}$, making most dimension eight operators irrelevant.

There are thousands of LFV dimension eight operators [17, 45], so it would be attractive to include only some of them in a first approximation. Indeed, in a bottom-up perspective, only the dimension eight operators to which observables are sensitive are required. So we reject derivative operators such as

$$
D^{\alpha}\left(\bar{e} \gamma_{\beta} \mu\right) D_{\alpha}\left(\bar{f} \gamma^{\beta} f\right)
$$

because their contribution to low-energy S-matrix elements should be suppressed by $\{s, t, u\} / v^{2}$, suggesting that $K$ and $\mu$ processes have no sensitivity to dimension eight derivative operators. We also neglect operators with more than four legs after electroweak symmetry-breaking, on the assumption that they do not contribute (at tree level) to our low-energy observables.

There remain about four dozen $\mu \leftrightarrow e$ operators (given in appendix A in the notation of $[17])$ :

1. four-particle operators which are forbidden at dimension 6 due to gauge invariance.

2. dimension six SMEFT operators with an additional $H$ and $H^{\dagger}$, such as $\left(H H^{\dagger}\right) \bar{\ell} H \sigma^{\alpha \beta} e F_{\alpha \beta}$ or $\left(\bar{\ell}_{e} H \sigma^{\alpha \beta} \mu\right)\left(\bar{q}_{i} \tilde{H} \sigma_{\alpha \beta} u_{j}\right)$. It may seem unlikely that the dimension eight contribution could be relevant given the possibility of a dimension six term ${ }^{5}$ however, being agnostic could be appropriate in EFT, and dimension eight operators are considered, for instance, in studies of Non-Standard neutrino Interactions [46].

These operators are schematically listed in tables 5 and 6 , along with the scale below they could contribute to observables with a coefficient $C \lesssim 1$. So they should be considered in the EFT parametrisation of any model constructed below this scale.

The effects of these operators can be partially accounted for by matching the model onto them at $\Lambda_{\mathrm{NP}}$, and then including them in the matching at the weak scale onto the low energy EFT. These matching conditions for LFV operators are given in appendix B (at tree level).

Many of these operators contribute to observables via loops, so including them in RGEs is relevant. Since they match at $m_{W}$ onto low-energy four-particle interactions, the Renormalisation Group running below $m_{W}$ is known and will occur automatically once they are included in the matching.

The RG running in SMEFT is missing. Above $m_{W}$, the Higgs and $W$ bosons can mix operators differently from the gluon and photon, for instance by modifying the SU(2) contractions (see eg the RGEs for a subset of dimension eight operators in [48]). Dimension eight four-fermion operators involving two tops pose a particular problem, because their

\footnotetext{
${ }^{5}$ The dimension six coefficient could perhaps be suppressed by additional loops or small couplings with respect to dimension eight.
} 


\begin{tabular}{|l|c|l|}
\hline operator & $\Lambda_{\mathrm{NP}}($ in TeV $)$ & process \\
\hline$\left(\bar{\ell}_{e} H e_{\mu}\right)\left(\bar{q}_{d} H d_{d}\right)$ & 55 & $\mu A \rightarrow e A$ \\
$\left(\bar{\ell}_{e} H e_{\mu}\right)\left(\bar{u}_{u} \tilde{H}^{\dagger} q_{u}\right)$ & 55 & $\mu A \rightarrow e A$ \\
$\left(\bar{\ell}_{e} H e_{\mu}\right)\left(\bar{q}_{s} H d_{s}\right)$ & 26 & $\mu A \rightarrow e A$ \\
$\left(\bar{\ell}_{e} H \sigma e_{\mu}\right)\left(\bar{q}_{d} H \sigma d_{d}\right)$ & 25 & $\mu A \rightarrow e A$ \\
$\left(\bar{\ell}_{e} H \sigma e_{\mu}\right)\left(\bar{q}_{b} H \sigma d_{b}\right)$ & 25 & $\mu A \rightarrow e A$ \\
$\left(\bar{\ell}_{e} H e_{\mu}\right) G G$ & 20 & $\mu A \rightarrow e A$ \\
$\left(\bar{\ell}_{e} H \sigma e_{\mu}\right)\left(\bar{\ell}_{\tau} H \sigma e_{\tau}\right)$ & 20 & $\mu \rightarrow e \gamma$ \\
$\left(\bar{\ell}_{e} H e_{\mu}\right)\left(\bar{\ell}_{e} H e_{e}\right)$ & 15 & $\mu \rightarrow e \bar{e} e$ \\
$\left(\bar{\ell}_{e} H e_{\mu}\right)\left(\bar{u}_{c} \tilde{H}^{\dagger} q_{c}\right)$ & 15 & $\mu A \rightarrow e A$ \\
$\left(\bar{\ell}_{e} H \sigma e_{\mu}\right)\left(\bar{q}_{s} H \sigma d_{s}\right)$ & 15 & $\mu A \rightarrow e A$ \\
$\left(\bar{\ell}_{e} H e_{\mu}\right)\left(\bar{u}_{t} \tilde{H}^{\dagger} q_{t}\right)$ & 10 & $\mu \rightarrow e \gamma$ \\
$\left(\bar{\ell}_{e} H e_{\mu}\right)\left(\bar{q}_{b} H d_{b}\right)$ & 10 & $\mu A \rightarrow e A$ \\
$\left(\bar{\ell}_{e} H e_{\mu}\right)\left(\bar{\ell}_{\mu} H e_{\mu}\right)$ & 8 & $\mu \rightarrow e \gamma$ \\
$\left(\bar{\ell}_{e} H e_{\mu}\right) F F$ & 3 & $\mu A \rightarrow e A$ \\
\hline
\end{tabular}

Table 5. Dimension eight operators which induce at low energy four-particle contact interactions that do not arise at dimension six. The operators are represented schematically in the first column, and the second column gives the scale $\Lambda_{\mathrm{NP}}$ up to which the process of the third column (with upcoming sensitivity) could probe coefficients $\lesssim 1$. (The estimate for $\left(\bar{\ell}_{e} H e_{\mu}\right) F F$ is from [47].) .

leading contribution to low energy LFV is likely to arise from the unknown RG running in SMEFT. Fortunately, many of these top operators are dimension six operators with an extra $H^{\dagger}$ and $H$ (only the operator $\sim\left(\bar{e} P_{R} \mu\right)\left(\bar{t} P_{L} t\right)$ arises first at dimension eight), so one could hope that models dominantly generate dimension six operators. Alternatively, one could envisage to add the coefficients of dimension eight top operators to the dimension six coefficients at $\Lambda_{\mathrm{NP}}$, and evolve them with the SMEFT RGEs at dimenson six, which will include a subset of the loops. We leave calculating the anomalous dimensions for a later project.

\subsection{2-loop anomalous dimensions?}

This section aims to identify relevant mixing that could arise from the two-loop RGEs of SMEFT, so we are looking for two-loop diagrams that would not be generated at second order in the one-loop RGEs.

One can see why these could be interesting, by considering the $\mathrm{QED} \times \mathrm{QCD}$-invariant EFT below $m_{W}$, where at one-loop, vector operators mix among themselves, and the dipoles+scalars+tensors mix among themselves, but there are no divergent one-loop diagrams mixing vectors and non-vectors. Therefore, to all orders in the one-loop RGEs, the vectors evolve separately from the others. However, vector to dipole mixing occurs at two-loop, and is encoded in the two-loop RGEs [49]; a few diagrams are given in figure 2. 


\begin{tabular}{|l|c|l|}
\hline operator & $\Lambda_{\mathrm{NP}}$ (in TeV) & process \\
\hline$\left(H^{\dagger} H\right)\left(\bar{\ell}_{e} \sigma e_{\mu}\right)\left(\bar{q}_{t} \sigma u_{t}\right)$ & 100 & $\mu \rightarrow e \gamma$ \\
$\left(H H^{\dagger}\right)\left(\bar{\ell}_{e} e_{\mu}\right)\left(\bar{d}_{d} q_{d}\right)$ & 55 & $\mu A \rightarrow e A$ \\
$\left(H H^{\dagger}\right)\left(\bar{\ell}_{e} e_{\mu}\right) \epsilon\left(\bar{q}_{u} u_{u}\right)$ & 55 & $\mu A \rightarrow e A$ \\
$\left(H H^{\dagger}\right)\left(\bar{\ell}_{e} e_{\mu}\right)\left(\bar{d}_{s} q_{s}\right)$ & 25 & $\mu A \rightarrow e A$ \\
$\left(H H^{\dagger}\right)\left(\bar{\ell}_{e} \gamma^{\alpha} \ell_{\mu}\right)\left(\bar{q}_{u} \gamma_{\alpha} q_{u}\right)$ & 22 & $\mu A \rightarrow e A$ \\
$\left(H H^{\dagger}\right)\left(\bar{\ell}_{e} \gamma^{\alpha} \ell_{\mu}\right)\left(\bar{u}_{u} \gamma_{\alpha} u_{u}\right)$ & 22 & $\mu A \rightarrow e A$ \\
$\left(H H^{\dagger}\right)\left(\bar{\ell}_{e} \gamma^{\alpha} \ell_{\mu}\right)\left(\bar{q}_{d} \gamma_{\alpha} q_{d}\right)$ & 22 & $\mu A \rightarrow e A$ \\
$\left(H H^{\dagger}\right)\left(\bar{\ell}_{e} \gamma^{\alpha} \ell_{\mu}\right)\left(\bar{d}_{d} \gamma_{\alpha} d_{d}\right)$ & 22 & $\mu A \rightarrow e A$ \\
$\left(H H^{\dagger}\right) \bar{\ell}_{e} H \sigma^{\alpha \beta} e_{\mu} F_{\alpha \beta}$ & 20 & $\mu \rightarrow e \gamma$ \\
$\left(H H^{\dagger}\right)\left(\bar{\ell}_{e} \gamma^{\alpha} \ell_{\mu}\right)\left(\bar{\ell}_{e} \gamma_{\alpha} \ell_{e}\right)$ & 18 & $\mu \rightarrow e \bar{e} e$ \\
$\left(H H^{\dagger}\right)\left(\bar{\ell}_{e} \gamma^{\alpha} \ell_{\mu}\right)\left(\bar{e}_{e} \gamma_{\alpha} e_{e}\right)$ & 18 & $\mu \rightarrow e \bar{e} e$ \\
$\left(H H^{\dagger}\right)\left(\bar{e}_{e} \gamma^{\alpha} e_{\mu}\right)\left(\bar{e}_{e} \gamma_{\alpha} e_{e}\right)$ & 18 & $\mu \rightarrow e \bar{e} e$ \\
$\left(H H^{\dagger}\right)\left(\bar{\ell}_{e} e_{\mu}\right) \epsilon\left(\bar{q}_{c} u_{c}\right)$ & 15 & $\mu A \rightarrow e A$ \\
$\left(H H^{\dagger}\right)\left(\bar{\ell}_{e} e_{\mu}\right)\left(\bar{d}_{b} q_{b}\right)$ & 10 & $\mu A \rightarrow e A$ \\
\hline
\end{tabular}

Table 6. Dimension eight operators which induce low energy contact interactions that do arise at dimension six. In the first column the operators are represented schematically (other distributions of the Higgses, or triplet constractions, could be possible), and the second column gives the scale $\Lambda_{\mathrm{NP}}$ up to which the process of the third column (with upcoming sensitivity) could probe coefficients $\lesssim 1$.

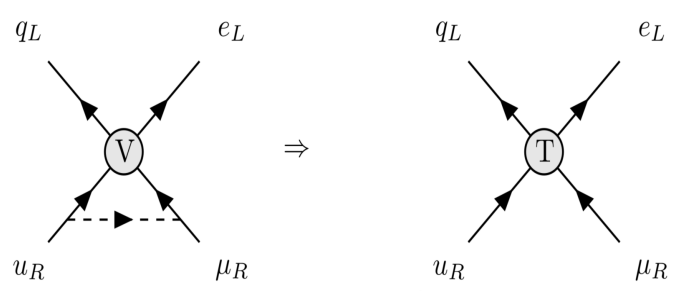

Figure 3. Vector mixing to the tensor via Higgs exchange.

So we are looking for two-loop diagrams that allow operator $\mathcal{O}$ to mediate process $\mathcal{P}$, when $\mathcal{O}$ cannot mediate $\mathcal{P}$ via the one-loop RGEs.

In SMEFT, there can be 1-loop vector to tensor mixing by exchanging a Higgs, as illustrated in figure 3. Closing the quark legs gives a contribution to the dipole. For instance, considering the vector $\mathcal{O}_{\ell q}^{(1)}$ we find

$$
\Delta C_{D} \sim e\left(\frac{\log }{16 \pi^{2}}\right)^{2} C_{\ell q}^{(1) e \mu n m}\left[Y_{u} Y_{u}^{\dagger}\right]_{n m} \frac{v^{2}}{\Lambda_{\mathrm{NP}}^{2}} \sim e \lambda^{4} C_{\ell q}^{(1) e \mu n m}\left[Y_{u} Y_{u}^{\dagger}\right]_{n m} \frac{v^{2}}{\Lambda_{\mathrm{NP}}^{2}}
$$

which results in a sensitivity to $C_{\ell q}^{(1) e \mu t t}$ up to $\Lambda_{\mathrm{NP}} \sim 50 \mathrm{TeV}$. Estimates similar to eq. (3.2) hold for all vector operators which can mix to the $u$-type tensor. 


\begin{tabular}{|c|c|c|}
\hline operator & 2loop $\mathrm{V} \rightarrow \mathrm{D}\left(\Lambda_{\mathrm{NP}}\right.$ in $\left.\mathrm{TeV}\right)$ & $\mathrm{V} \rightarrow \mathrm{T} \rightarrow \mathrm{D}\left(\Lambda_{\mathrm{NP}}\right.$ in $\left.\mathrm{TeV}\right)$ \\
\hline $\mathcal{O}_{\ell q}^{(1) e \mu t t}$ & 10 & 50 \\
\hline $\mathcal{O}_{V}^{e \mu f f}$ & 10 & \\
\hline $\mathcal{O}_{\ell e d q}^{e \mu d d}$ & 5 & - \\
\hline $\mathcal{O}_{\text {ledq }}^{\text {epss }}$ & 20 & - \\
\hline $\mathcal{O}_{\ell e d q}^{e \mu b b}$ & 100 & - \\
\hline
\end{tabular}

Table 7. Operators which contribute to $\mu \rightarrow e \gamma$ via two-loop mixing in the RGEs, and in the second column, our powercounting estimate for the scale $\Lambda_{\mathrm{NP}}$ up to which coefficients $\lesssim 1$ could be probed. The third column gives the estimated sensitivity obtainable via the one-loop RGEs. $\mathcal{O}_{V}^{\text {enff }}$ schematically refers to all the dimension six vector four-fermion operators with $f \neq t$. .

Vector operators also can mix directly to the dipole in the 2-loop RGEs through gauge interactions, as illustrated by the diagram on the left of figure 2 . The powercounting estimate for these diagrams

$$
\Delta C_{D} \sim e^{3} \frac{\log }{\left(16 \pi^{2}\right)^{2}} C_{V} \frac{v^{2}}{\Lambda_{\mathrm{NP}}^{2}}
$$

suggests that there is sensitivity to vector coefficients for scales below $10 \mathrm{TeV}$ - which is larger than the vector $\rightarrow$ tensor $\rightarrow$ dipole contribution for all operators not involving a top quark, see table 7 .

There could also be two-loop mixing of the $\mathcal{O}_{L E D Q}$ scalar to the dipoles. For comparaison, at one loop the $u$ quark scalar operator $\mathcal{O}_{L E Q U}$ mixes to the tensor, which mixes to the dipole, and due to Yukawa enhancement and large anomalous dimensions, this second-order process in the one-loop RGEs is important. In the $d$-quark sector, there is no dimension six tensor, so no equivalent process occurs; however the diagrams are there, and $\mathcal{O}_{L E D Q}$ can be Fierzed to the vector $-\frac{1}{2}\left(\bar{\ell} \gamma_{\alpha} q\right)\left(\bar{d} \gamma_{\alpha} e\right)$ which mixes at two-loop to the dipole [49]. The powercounting estimate is

$$
\Delta C_{D} \sim e g^{2} \lambda^{5} C_{l e d q}^{i j}\left[Y_{d}\right]_{i j} \frac{v^{2}}{\Lambda_{\mathrm{NP}}^{2}}
$$

which suggests that $\mu \rightarrow e \gamma$ could be sensitive to coefficients $\lesssim 1$ up to the scales given in table 7 .

These results show that the two-loop vector to dipole mixing can be relevant, and often dominates over the mixing involving a Higgs loop, which occurs at second-order in the one-loop RGEs. It would be desirable to include these two-loop anomalous dimensions. However, although they are known in QCD and QED [11, 49, 50], a complete computation in SMEFT is currently missing in the literature [51].

\section{$3.3 \quad$ CKM}

CKM mixing angles can appear in various places in SMEFT: in matching of the higher scale theory onto SMEFT, in the RG running of operator coefficients and of SM couplings, and in 


\begin{tabular}{|l|c|c|c|c|c|c|}
\hline$i j \backslash p r$ & $\mathrm{bb}$ & $\mathrm{bs}$ & $\mathrm{bd}$ & $\mathrm{ss}$ & $\mathrm{sd}$ & $\mathrm{dd}$ \\
\hline $\mathrm{tt}$ & $\lambda^{5}$ & $\lambda^{9.5}$ & $\lambda^{13}$ & $\lambda^{14}$ & $\lambda^{17.5}$ & $\lambda^{21}$ \\
$\mathrm{tc}$ & $\lambda^{7}$ & $\lambda^{7.5}$ & $\lambda^{10.5}$ & $\lambda^{12}$ & $\lambda^{15}$ & $\lambda^{18.5}$ \\
$\mathrm{tu}$ & $\lambda^{8.5}$ & $\lambda^{8.5}$ & $\lambda^{9.5}$ & $\lambda^{13}$ & $\lambda^{14}$ & $\lambda^{17.5}$ \\
$\mathrm{cc}$ & $\lambda^{9}$ & $\lambda^{9.5}$ & $\lambda^{12.5}$ & $\lambda^{10}$ & $\lambda^{13}$ & $\lambda^{16}$ \\
$\mathrm{cu}$ & $\lambda^{10.5}$ & $\lambda^{10.5}$ & $\lambda^{11.5}$ & $\lambda^{11}$ & $\lambda^{12}$ & $\lambda^{15}$ \\
$\mathrm{uu}$ & $\lambda^{12}$ & $\lambda^{12}$ & $\lambda^{13}$ & $\lambda^{12}$ & $\lambda^{13}$ & $\lambda^{14}$ \\
\hline
\end{tabular}

Table 8. Estimates for the Yukawa and CKM suppression $\left(\simeq V_{C K M}^{i p} y_{d_{p}} V_{C K M}^{j r} y_{d_{r}}\right)$ of the mixing between operators containing $\left(\bar{q}_{i} \gamma_{\alpha} q_{j}\right)$ into operators containing $\left(\bar{d}_{p} \gamma_{\alpha} d_{r}\right)$. The indices $i j$ are given in the left column, and $p r$ in the top line.

matching the SMEFT operators at $m_{W}$ onto the $\mathrm{QED} \times \mathrm{QCD}$-invariant low energy theory. Including CKM in matching at $m_{W}$ is straightforward, but it could be conceptually simpler to set $V_{C K M}=1$ in the RGEs for the operator coefficients. This section explores the errors that could arise from this approximation, by allowing one non-zero operator at a time at $\Lambda_{\mathrm{NP}}$, and estimating the magnitude of low-energy coefficients that it generates at one-loop $\propto\left[V_{C K M}\right]_{i j}, i \neq j$. If no experiment has sensitivity to the contributions proportional to CKM mixing angles, then one can conclude that $V_{C K M}=1$ is an acceptable approximation in the RGEs.

The CKM matrix also appears in the RGEs of the renormalisable SM couplings, where it causes the eigenbases of $Y_{d} Y_{d}^{\dagger}$ and $Y_{u} Y_{u}^{\dagger}$ to rotate with scale. This is due to wavefunction corrections. Since wavefunction diagrams also decorate the operators, we assume this is a "universal" effect, automatically included by working in the rotating $Y_{u} Y_{u}^{\dagger}$ eigenbasis, and do not powercount the associated diagrams. ${ }^{6}$

Recall that we work in the $Y_{d}$ eigenbasis for the $\left\{d_{R}\right\}$, and the $Y_{u}$ eigenbasis for the $\left\{u_{R}\right\}$ and $\left\{q_{L}\right\}$. So $V_{C K M}$ only appears in Higgs loops, at vertices $\propto Y_{d}=V_{C K M} D_{d}$. It therefore enters the one-loop RGEs of $\mathcal{O}_{L Q 1}, \mathcal{O}_{L Q 3}, \mathcal{O}_{L D}, \mathcal{O}_{E D} \mathcal{O}_{E Q}$ and $\mathcal{O}_{L E D Q}$.

Consider first operators at $\Lambda_{\mathrm{NP}}$ with a doublet quark bilinear $\left(\bar{q}_{i} \gamma_{\alpha} q_{j}\right)$, where $i, j \in$ $\{u, c, t\}$. Higgs exchange between the quark legs can dress this quark bilinear to generate

$$
\left(\bar{q}_{i} \gamma_{\alpha} q_{j}\right) \rightarrow V_{C K M}^{i p} y_{d_{p}} V_{C K M}^{j r} y_{d_{r}} \frac{\log }{16 \pi^{2}}\left(\bar{d}_{p} \gamma_{\alpha} d_{r}\right)
$$

where the approximate magnitude of $V_{C K M}^{i p} y_{d_{p}} V_{C K M}^{j r} y_{d_{r}}$, for all possible flavours of the doublet and singlet lines, is given in table 8. If the CKM matrix is approximated as the identity, then only the diagonal components of the table would remain.

From the table 8 , one sees that mixing induced by non-vanishing CKM angles is suppressed by $<\lambda^{7+2} v^{2} / \Lambda_{\mathrm{NP}}^{2}$ (where the additional $\lambda^{2}$ is for the $\log / 16 \pi^{2}$ loop suppression). Such contributions are clearly negligeable in the RGEs for $\tau \rightarrow \ell$ operators; to determine

\footnotetext{
${ }^{6}$ For instance, an off-diagonal $\left[Y_{u}\right]^{c t} \sim 3 \log /\left(32 \pi^{2}\right) V_{c b} y_{b}^{2} V_{t b} y_{t}$ is generated by a Higgs loop on the $q_{L}$ line. Inside the loop mixing $\mathcal{O}_{L E Q U, 3} \rightarrow \mathcal{O}_{D}$, this could give sensitivity to $\mathcal{O}_{L E Q U, 3}^{e \mu c t}$, in an unrotating basis for $q_{L}$.
} 
whether they should be included in the RGEs for $\mu \leftrightarrow e$ operators, we compare to the sensitivity of upcoming experiments. In the case of $p=r$ but $i \neq j$, the best sensitivity is from $\mu \rightarrow e$ conversion. We estimate that $\mu A \rightarrow e A$ could be sensitive to the mixing from $\left(\bar{q}_{t} \gamma_{\alpha} q_{c}\right) \rightarrow\left(\bar{b} \gamma_{\alpha} P_{R} b\right)$ for an experimental reach $\mathrm{BR}(\mu A \rightarrow e A) \lesssim 10^{-16} \frac{v^{4}}{\Lambda_{\mathrm{NP}}^{4}}$, and to the $c u \rightarrow s s, d d$ mixing for $\operatorname{BR}(\mu A \rightarrow e A) \lesssim 10^{-20} \frac{v^{4}}{\Lambda_{\mathrm{NP}}^{4}}$. This suggests that the RGE-mixing of operators involving $\left(\bar{q}_{i} \gamma_{\alpha} q_{j}\right)$, into operators involving $\left(\bar{d}_{p} \gamma_{\alpha} d_{p}\right)$, for $i \neq j$ and $p=q$, is negligeable in the forseeable future. In the converse case, of RGE-mixing of flavour-diagonal operators $\left(\bar{q}_{i} \gamma_{\alpha} q_{i}\right)$, into quark flavour non-diagonal operators $\left(\bar{d}_{p} \gamma_{\alpha} d_{r}\right)$, table 8 indicates that the least suppressed mixings are $t t, c c \rightarrow b s \propto \lambda^{9.5} \frac{v^{2}}{\Lambda_{\mathrm{NP}}^{2}}$, and $c c, u u \rightarrow s d \propto \lambda^{13} \frac{v^{2}}{\Lambda_{\mathrm{NP}}^{2}}$, which is beyond the sensitivity of the meson decay searches listed in table 3 .

The CKM angles can also enter in the mixing of the singlet quark current $\left(\bar{d}_{p} \gamma_{\alpha} d_{p}\right)$ into doublets $\left(\bar{q}_{i} \gamma_{\alpha} q_{j}\right)$. Similarly to the doublet to singlet mixing discussed above, the effects of CKM are beyond upcoming experimental sensitivities. A novel feature in this case is that approximating the CKM angles to vanish can generate flavour change when there is none. For example, the $s_{R}$ leg of an operator could transform under RG running into a left-handed doublet quark (due to Higgs exchange), which in the SM would be in the $s_{L}$ direction. But in our approximation where $Y_{d} Y_{d}^{\dagger}$ is diagonal in the $Y_{u} Y_{u}^{\dagger}$ eigenbasis, it is in the $c_{L}$ direction, so matches at $m_{W}$ onto $\sum_{p} V_{C K M}^{c p} d_{L p}$.

Finally, there are diagrams with one Higgs vertex on the quark line and one on a lepton line, which eg mix vector and scalar operators. The mixing from scalar into vector operators, such as $\mathcal{O}_{L E D Q} \rightarrow\left\{\mathcal{O}_{L Q}^{(1)}, \mathcal{O}_{E Q}\right\}$ can be neglected because the lepton Yukawas are smaller than that of the $b$, so any quark-flavour-changing contribution is more suppressed than the $\left(\bar{d}_{p} \gamma_{\alpha} d_{p}\right) \leftrightarrow\left(\bar{q}_{i} \gamma_{\alpha} q_{j}\right)$ mixing discussed above. It is also the case that quark-flavourchanging mixing from vectors to scalars is below the sensitivity of upcoming experiments, despite that the experimental sensitivity to scalar operators can be better than to vectors (see table 3). In the case of $\mu \leftrightarrow e$ searches, this is because the mixing is suppressed by $y_{\mu} \sim \lambda^{5}$, and for $\tau \leftrightarrow \ell$ searches, the experiments are less sensitive.

So we conclude that CKM angles can be neglected in the SMEFT RGEs for LFV operators, provided that one runs in the $Y_{u} Y_{u}^{\dagger}$ eigenbasis for the $\left\{q_{L}\right\}$, and that CKM mixing is retained in matching at $m_{W}$.

\subsection{LFV Yukawa couplings}

In the SM, the Yukawa matrix of the charged leptons is the only basis-choosing interaction in the leptonic sector - the gauge interactions are "universal", that is, proportional to the identity matrix in generation space, so the eigenvectors do not choose directions. In the real world (not described by the SM), the neutrino mass matrix provides another eigenbasis, but the magnitude of neutrino masses is so small that their direct GIM-suppressed contribution to LFV is irrelevant (instead, they provide motivation to search for LFV).

LFV operators that are added to the Lagrangian below the weak scale are inevitably written in the mass eigenstate basis of the charged leptons. Above the weak scale in SMEFT, there are two possibilities: the mass eigenstate basis, or the Yukawa eigenstate basis - which may be different in the presence of the operator $\mathcal{O}_{e H}$. The physics, of course, 
cannot depend on a basis choice, but the calculation may be more intuitive and simple in somes bases than in others. So which is the best choice?

Suppose one thinks top-down; then at $\Lambda_{\mathrm{NP}}$, the New Physics model is matched to the SM +operators. The obvious basis in this case for SMEFT is the $D_{e}$-basis where the lepton Yukawa matrix is diagonal: $Y_{e}=D_{e}=\operatorname{diag}\left\{y_{e}, y_{\mu}, y_{\tau}\right\}$. This choice is motivated by LFV being a NP effect, and ensures that the SMEFT RGEs, which describe SM dynamics, cannot change the flavours of lepton legs.

However, when the Higgs gets a vev in the presence of the $\mathcal{O}_{e H}$ operator, the $D_{e}$ basis may no longer be the mass eigenstate basis, due to additional off-diagonal contributions of $\mathcal{O}_{e H}$ to the mass matrix. So a basis rotation during the matching at $m_{W}$ would be required, from the $D_{e}$ basis to the mass eigenstate basis in which the restrictive low-energy constraints are expressed. Current constraints/sensitivities on the off-diagonal elements of $\mathcal{O}_{e H}$ imply that the angles of this rotation are small: estimating $\theta_{i j} \sim C_{e H}^{i j} v^{3} /\left(\Lambda_{\mathrm{NP}}^{2} \max \left\{m_{i}, m_{j}\right\}\right)$ for $i \neq j$ gives

$$
\theta_{\ell \tau}, \theta_{\tau \ell} \lesssim \lambda, \quad \theta_{e \mu}, \theta_{\mu e} \lesssim \lambda^{4}
$$

where $\ell \in\{e, \mu\}$.

If the New Physics scale is sufficiently high that only dimension six operators are relevant, one might hope to neglect this rotation in matching, because the angles are $\propto C v^{2} / \Lambda_{\mathrm{NP}}^{2}$, so any effect on a NP operator would be $\mathcal{O}\left(1 / \Lambda_{\mathrm{NP}}^{4}\right)$. (Below $m_{W}$, there are also contact interactions induces by the $W, Z, h$, which could becomes flavour-changing under a basis rotation. However, the $W$ and $Z$ interactions are "universal", so unconcerned by basis rotations, and the higgs-mediated operators are suppressed by SM Yukawas, so the dimension six flavour-changing operators induced by the rotation are unobservable.) However, as previously discussed, LFV data can have sensitivity to operators suppressed by $\mathcal{O}\left(1 / \Lambda_{\mathrm{NP}}^{4}\right)$, and the mixing angles of eq. (3.6) are also enhanced by inverse Yukawas. The power-counting rules suggest that flavour-diagonal coefficients at $\Lambda_{\mathrm{NP}} \sim 4 \mathrm{TeV}$ could be rotated into $\tau \leftrightarrow \ell$ operators suppressed by $\lambda^{5}$, and into $\mu \leftrightarrow e$ suppressed by $\lambda^{8}$. This is within current experimental sensitivities.

We advocate not making the transformation from the mass to Yukawa eigenstate basis at $m_{W}$. This is because the rotation is unknown, and the angles are insufficiently suppressed (see eq. (3.6)). Instead, we remain in the mass eigenstate basis above the weak scale; this is consistent with our bottom-up perspective, because it is the basis where the constraints apply. The lepton Yukawa matrix can be off-diagonal in this basis(see eq. (2.9)), but the off-diagonals $\sim \theta_{i j} y_{j}$ are much smaller than the $\theta_{i j}$ s of eq. (3.6) because they are suppressed also by small lepton Yukawas. The powercounting suggests that they can be neglected in the RGEs, for instance

$$
\left[Y_{e}\right]_{\mu e} \frac{\log }{16 \pi^{2}} \frac{v^{2}}{\Lambda_{\mathrm{NP}}^{2}} \lesssim \lambda^{15}
$$

So in practise, we work in the mass eigenstate basis at all scales, but treat the lepton Yukawa matrix as diagonal in the RGEs of SMEFT. The inconvenience of this choice is that in matching a model onto the operators, one must identify the low energy lepton mass eigenstate basis in the model, and obtain operator coefficients in that basis. 


\subsection{LFV with $\tau$ s}

This section briefly discusses the ingredients required for a "leading order" SMEFT study of LFV involving $\tau \mathrm{s}$.

For the majority of $\tau$ LFV processes listed in table 3 there is sensitivity to Wilson coefficients that are $\gtrsim \lambda^{5}$. Since a loop costs a factor $\lambda^{2}$, loop effects in the $\tau$ sector could be relevant for $\left(v^{2} / \Lambda_{\mathrm{NP}}^{2}\right) \geq \lambda^{3}$, but this implies a New Physics scale within the LHC reach.

In the case of the more sensitive $\tau \rightarrow e(\mu) \gamma$ searches, the corresponding diagrams can be power counted as for $\mu \rightarrow e \gamma$, replacing the muon leg with a tau leg. Since the constraints concern dipole coefficients defined with a built-in yukawa of the heavier lepton, we encounter two possibilities in the diagrams:

- either one Higgs leg is attached to the decaying lepton line and the power counting estimate is the same,

- or no Higgs-heavy lepton vertex is present and the diagrams are suppressed by a factor $y_{\mu} / y_{\tau}=2 \lambda^{2}$ with respect to the corresponding $\mu \rightarrow e \gamma$ one.

In both cases, given the lesser sensitivity in the $\tau$ sector, we can conclude that any approximation that we justify through power counting for $\mu$-s is also valid for $\tau$ LFV processes.

As a result, two-loop anomalous dimensions should be irrelevant in $\tau \leftrightarrow \ell$ processes, due to the estimated suppression $\sim \lambda^{5}$ of two-loop diagrams. This should remain true even in the case of $\tau \rightarrow e(\mu) \gamma$.

Furthermore, the requirement of eq. (3.1) on 8-dimensional operator coefficients for $\Lambda_{\mathrm{NP}} \gtrsim 4 \mathrm{TeV}$

$$
{ }^{(8)} C \lesssim \lambda^{8}
$$

is sufficient to argue that any $\tau$ LFV observable is not sensitive to dimension eight operators.

\section{Summary}

Effective Field Theory can be envisaged from a bottom-up or top-down perspective. In bottom-up EFT for Lepton Flavour Change(LFV), the aim is to map experimental constraints onto the correct sum of operator coefficients at the New Physics scale $\Lambda_{\mathrm{NP}}$, in order to identify the area in coefficient space where BSM models must sit. From a top-down perspective, one can map a LFV model onto operator coefficients at $\Lambda_{\mathrm{NP}}$, calculate observables using EFT, and this should correctly reproduce model predictions to within a calculable uncertainty. In both perspectives, the EFT calculation must include correctly every operator coefficient that could contribute to an observable, irrespective of its dimension or of the order in the loop or coupling expansions.

To ensure that we use SMEFT correctly for describing LFV, we introduced a powercounting scheme, that allows to organise all the SMEFT perturbative expansions - in loops, couplings, mixing angles and the ratio of the weak scale to the New Physics $v / \Lambda_{\mathrm{NP}}$ — in terms of a small "Cabibbo-Wolfenstein-like" parameter $\lambda \approx 0.2$. This power-counting scheme is described in section 2.2 , and summarised in table 2 . The future reach of various 
experiments can be expressed in powers of $\lambda$ (see table 3) - so for instance, the upcoming MEGII experiment searching for $\mu \rightarrow e \gamma$ could probe dipole coefficients up to $\mathcal{O}\left(\lambda^{12}\right)$. Then one can draw diagrams, arising at various orders in the different perturbative expansions, and do two things; first, compare different contributions of an operator to an observable, to identify the leading one, (see eg section 2.3 and 3.2). And secondly, one can determine which operators can affect which observables by comparing the power-counting estimates to the future experimental sensitivity. Some examples are given in section 2.3.

For LFV operators, the SMEFT expansion in operator dimension can be written as an expansion in $v^{2} / \Lambda_{\mathrm{NP}}^{2}$, where the New Physics scale $\Lambda_{\mathrm{NP}}$ plays two roles in our manuscript. On one hand, it is the unknown mass of the lightest lepton flavour changing new particle (see the Lagrangian of eq. (2.1)), which we take "beyond the reach of the LHC": $\Lambda_{\mathrm{NP}} \gtrsim 4 \mathrm{TeV}$ (so $v^{2} / \Lambda_{\mathrm{NP}}^{2} \lesssim \mathcal{O}\left(\lambda^{4}\right)$ in the powercounting scheme). However, since $\Lambda_{\mathrm{NP}}$ is unknown, we simultaneously count the order of an operators contribution by the scale it could probe with a coefficient of $\mathcal{O}\left(1 / \Lambda_{\mathrm{NP}}^{2 n}\right)$.

In the SMEFT, there are already many operators at dimension six, and their RGEs are only known at one-loop. So in section 3, we use the powercounting scheme to explore whether dimension six operators and one-loop RGEs are sufficient to describe LFV at the sensitivity of experiments under construction. Section 3.2 suggests that some two-loop anomalous dimensions are required for $\mu \leftrightarrow e$ flavour change, when $\Lambda_{\mathrm{NP}} \lesssim 20 \mathrm{TeV}$. The calculation of these anomalous dimensions is in progress [51].

Section 3.1 finds that upcoming $\mu \leftrightarrow e$ data can be sensitive to dimension eight SMEFT operators, about four dozen of them for $\Lambda_{\mathrm{NP}} \gtrsim 4 \mathrm{TeV}$, but none at scales $\Lambda_{\mathrm{NP}} \gtrsim 100 \mathrm{TeV}$. The relevant dimension eight operators match onto three-or four-point interactions below the weak scale, and can be divided into two sets: those which are the lowest-dimension SMEFT operator inducing a given contact interaction below $m_{W}$, and a second set that induces low-energy contact interactions already present at dimension six. The scale $\Lambda_{\mathrm{NP}}$ up to which the operators can be relevant is given in tables 5 and 6 . These dimension eight operators are listed in appendix A, and are included in the matching onto operators below $m_{W}$ in appendix B.

The power counting scheme can also be used to simplify and streamline calculations with the existing SMEFT operators and RGEs, for instance by neglecting flavour-changing SM interactions. We perform two such exercises; section 3.3 checks that CKM mixing can be neglected in the RGEs for LFV operators, provided that it is included in matching, and that the SMEFT RGEs run in the $Y_{u} Y_{u}^{\dagger}$ eigenbasis for the $\left\{q_{L}\right\}$. Section 3.4 explores the case where operators of the form $C^{i j}\left(H^{\dagger} H\right)^{n} \bar{\ell}_{i} H e_{j}$, with $i \neq j$, are allowed to contribute to the charged lepton mass matrix. This implies that in the charged lepton mass eigenstate basis (where all experimental constraints are given), the charged lepton Yukawa $Y_{e}$ has unknown off-diagonal elements. The power-counting suggests that if these flavourchanging Yukawas are below current experimental sensitivities, they can be neglected in the SMEFT RGEs.

In this manuscript, we estimated lower bounds on the scale $\Lambda_{N P}$, such that the predictions of lepton flavour changing New Physics models from beyond $\Lambda_{N P}$ can be obtained with the dimension six operators of SMEFT and their one-loop RGEs. These results could 
be used to motivate, or justify, SMEFT studies of LFV. It could be interesting to perform a similar study in the EFT with a "non-linear realisation" of the Higgs sector [52-55], and also to perform a more systematic expansion to ensure that the leading terms are identified.

\section{Acknowledgments}

We thank Junji Hisano for proposing the initial powercounting scheme, and Luca Silvestrini for useful conversations. MA is supported by a doctoral fellowship from the IN2P3.

\section{A Some LFV Operators of dimension eight}

Section 3.1 showed that $\mu \leftrightarrow e$ processes can be sensitive to some SMEFT operators of dimension eight, if these have $\mathrm{O}(1)$ coefficients at $\Lambda_{\mathrm{NP}} \gtrsim 4 \mathrm{TeV}$. This appendix lists the relevant operators, following the notation of [17].

The LFV operators given here are required to match onto low energy operators involved in the processes of table 3, so derivative operators, and those involving more than four particles at low energy, are neglected. In addition, operators of the form $\mu_{H}^{2} \times$ dimension six, where $\mu_{H}^{2}$ is the Higgs mass ${ }^{2}$ term in the Lagrangian, are neglected because in matching onto operators below $m_{W}$, the potential minimisation condition relates $\mu_{H}^{2}$ to $H^{\dagger} H$. Furthermore, we restrict our list to operators that are $\mu \leftrightarrow e$ flavour changing but flavour diagonal in the two other fermion legs, as the low energy observables constrain operator with this flavour structure.

The four-fermion operators of dimension eight can be obtained by adding two Higgs fields to dimension six four-fermion operators, or by multiplying two renormalizable Lagrangian terms. Dimension six operators can be multiplied by the singlet product $\left(H^{\dagger} H\right)$, but the Higgses can also contract with specific doublets; when the Higgs gets a vev, this feature induces a low-energy operator involving only some $\mathrm{SU}(2)$ partners. For instance, the dimension eight operator

$$
\left(\bar{\ell}_{\alpha} \tilde{H} \gamma_{\rho} \tilde{H}^{\dagger} \ell_{\beta}\right)\left(\bar{q} \gamma^{\rho} q\right) \rightarrow\left(\bar{\nu}_{\alpha} \gamma_{\rho} \nu_{\beta}\right)\left(\bar{u} \gamma^{\rho} u+\bar{d} \gamma^{\rho} d\right) .
$$

This operator induces "Non-Standard neutrino Interactions" [46], which can be searched for at neutrino experiments, without inducing tree-level flavour-change among charged leptons. Exploiting $\mathrm{SU}(2)$ identities, these operators can be expressed as linear combinations of $\operatorname{dim} 6 \times\left(H^{\dagger} H\right)$ and the following operator

$$
\left(\bar{\ell}_{\alpha} \tau^{I} \gamma_{\rho} \ell_{\beta}\right)\left(\bar{q} \gamma^{\rho} q\right)\left(H^{\dagger} \tau^{I} H\right)
$$

Adopting the convention of [17], we retain the triplet contractions in the operator basis. Since we are interested in the contribution of dimension eight operators to LFV observables, we organize the operator list according to whether a dimension six version exists or does not exist.

We display operators with "standard" flavour indices and we don't include the permutations that will be matched to the same low energy interaction, as discussed in appendix B. 


\section{A.1 Dimension eight not present at dimension six}

\section{A.1.1 Four-fermion}

$\mathrm{SU}(2)$ invariance and its chiral nature forbid SMEFT dimension six counterparts of some four-fermion contact interaction of the $\mathrm{QCD} * \mathrm{QED}$ invariant Lagrangian, forcing their appearance at dimension eight. In the case of four-fermion operators with four-lepton legs these are the tensor operators

$$
\mathcal{O}_{L^{2} E^{2} H^{2}}^{(4) e \mu k}=\left(\bar{l}_{e} H \sigma^{\alpha \beta} e_{\mu}\right)\left(\bar{l}_{k} H \sigma_{\alpha \beta} e_{k}\right)
$$

where $k \in\{e, \mu, \tau\}$. They can be related to the scalars $\mathcal{O}_{L^{2} E^{2} H^{2}}^{(3) i k l}=\left(\bar{l}_{i} H e_{j}\right)\left(\bar{l}_{k} H e_{l}\right)$ of the basis [17] thanks to the following Fierz identity

$$
\mathcal{O}_{L^{2} E^{2} H^{2}}^{(4) e \mu k k}=-8 \mathcal{O}_{L^{2} E^{2} H^{2}}^{(3) k k \mu}-4 \mathcal{O}_{L^{2} E^{2} H^{2}}^{(3) e \mu k k}
$$

Given that the tensors mix with the dipole, we retain both operators in the matching conditions of appendix B, keeping in mind that we can remove the redundancy by means of the above identity.

For four-fermion interaction involving two-lepton and two-quark legs, the dimension eight operators that do not arise at dimension six are

$$
\begin{array}{ll}
\mathcal{O}_{L E D Q H^{2}}^{(3) e \mu n n} & =\left(\bar{\ell}_{e} H e_{\mu}\right)\left(\bar{q}_{n} H d_{n}\right) \quad \mathcal{O}_{L E D Q H^{2}}^{(4) e \mu n n}=\left(\bar{\ell}_{e} \sigma^{\alpha \beta} H e_{\mu}\right)\left(\bar{q}_{n} \sigma_{\alpha \beta} H d_{n}\right) \\
\mathcal{O}_{L E Q U H^{2}}^{(5) e \mu n n} & =\left(\bar{\ell}_{e} H e_{\mu}\right)\left(\bar{u}_{n} \tilde{H}^{\dagger} q_{n}\right) .
\end{array}
$$

where $n$ is a quark generation index. In this case, the scalar and tensor operator for down-type quarks are independent and cannot be related by means of Fierz identities.

\section{A.1.2 Two-lepton operators}

Two-lepton and two-gauge boson operators firstly appear at dimension eight

$$
\begin{aligned}
\mathcal{O}_{L E G^{2} H}^{(1) e \mu} & =\left(\bar{\ell}_{e} H e_{\mu}\right) G_{\alpha \beta}^{A} G^{A \alpha \beta} & \mathcal{O}_{L E G^{2} H}^{(2) e \mu} & =\left(\bar{\ell}_{e} H e_{\mu}\right) G_{\alpha \beta}^{A} \tilde{G}^{A \alpha \beta} \\
\mathcal{O}_{L E W^{2} H}^{(1) e \mu} & =\left(\bar{\ell}_{e} H e_{\mu}\right) W_{\alpha \beta}^{I} W^{I \alpha \beta} & \mathcal{O}_{L E W^{2} H}^{(2) e \mu} & =\left(\bar{\ell}_{e} H e_{\mu}\right) W_{\alpha \beta}^{I} \tilde{W}^{I \alpha \beta} \\
\mathcal{O}_{L E B^{2} H}^{(1) e \mu} & =\left(\bar{\ell}_{e} H e_{\mu}\right) B_{\alpha \beta} B^{\alpha \beta} & \mathcal{O}_{L E B^{2} H}^{(2) e \mu} & =\left(\bar{\ell}_{e} H e_{\mu}\right) B_{\alpha \beta} \tilde{B}^{\alpha \beta} \\
\mathcal{O}_{L E W B H}^{(1) e \mu} & =\left(\bar{\ell}_{e} \tau^{I} H e_{\mu}\right) B^{\alpha \beta} W_{\alpha \beta}^{I} & \mathcal{O}_{L E W B H}^{(2) e \mu} & =\left(\bar{\ell}_{e} \tau^{I} H e_{\mu}\right) B_{\alpha \beta} \tilde{W}^{I \alpha \beta}
\end{aligned}
$$

and provide the leading order matching contribution to the dimension seven twophoton $\mathcal{O}_{F F, Y}=\left(\bar{e} P_{Y} \mu\right) F_{\alpha \beta} F^{\alpha \beta}, \mathcal{O}_{F \tilde{F}, Y}=\left(\bar{e} P_{Y} \mu\right) F_{\alpha \beta} \tilde{F}^{\alpha \beta}$ and two-gluon $\mathcal{O}_{G G, Y}=$ $\left(\bar{e} P_{Y} \mu\right) G_{\alpha \beta}^{A} G^{A \alpha \beta}, \mathcal{O}_{G \tilde{G}, Y}=\left(\bar{e} P_{Y} \mu\right) G_{\alpha \beta}^{A} \tilde{G}^{A \alpha \beta}$ operators of the low energy Lagrangian, whose coefficients are constrained by searches of $\mu \rightarrow e$ conversion in nuclei. 


\section{A.2 Dimension eight operators present at dimension six}

\section{A.2.1 Four-fermion}

The four-fermion operators with four lepton legs that also appear at dimension six are

$$
\begin{array}{rlrl}
\mathcal{O}_{L^{4} H^{2}}^{(1) e \mu k k} & =\left(\bar{\ell}_{e} \gamma^{\alpha} \ell_{\mu}\right)\left(\bar{\ell}_{k} \gamma_{\alpha} \ell_{k}\right)\left(H^{\dagger} H\right) & \mathcal{O}_{L^{4} H^{2}}^{(2) e \mu k}=\left(\bar{\ell}_{e} \gamma^{\alpha} \ell_{\mu}\right)\left(\bar{\ell}_{k} \tau^{I} \gamma_{\alpha} \ell_{k}\right)\left(H^{\dagger} \tau^{I} H\right) \\
\mathcal{O}_{L^{2} E^{2} H^{2}}^{(1) \mu k k} & =\left(\bar{\ell}_{e} \gamma^{\alpha} \ell_{\mu}\right)\left(\bar{e}_{k} \gamma_{\alpha} e_{k}\right)\left(H^{\dagger} H\right) & \mathcal{O}_{L^{2} E^{2} H^{2}}^{(2) e \mu k k} & =\left(\bar{\ell}_{e} \tau^{I} \gamma^{\alpha} \ell_{\mu}\right)\left(\bar{e}_{k} \gamma_{\alpha} e_{k}\right)\left(H^{\dagger} \tau^{I} H\right) \\
\mathcal{O}_{E^{4} H^{2}}^{e \mu k k} & =\left(\bar{e}_{e} \gamma^{\alpha} e_{\mu}\right)\left(\bar{e}_{k} \gamma_{\alpha} e_{k}\right)\left(H^{\dagger} H\right), & &
\end{array}
$$

where $k=e, \mu, \tau$.

In addition, the four-fermion operators containing two-lepton and two-quark legs are:

$$
\begin{aligned}
& \mathcal{O}_{L^{2} Q^{2} H^{2}}^{(1) \mu n n}=\left(\bar{\ell}_{e} \gamma^{\alpha} \ell_{\mu}\right)\left(\bar{q}_{n} \gamma_{\alpha} q_{n}\right)\left(H^{\dagger} H\right) \\
& \mathcal{O}_{L^{2} Q^{2} H^{2}}^{(2) e \mu n n}=\left(\bar{\ell}_{e} \tau^{I} \gamma^{\alpha} \ell_{\mu}\right)\left(\bar{q}_{n} \gamma_{\alpha} q_{n}\right)\left(H^{\dagger} \tau^{I} H\right) \\
& \mathcal{O}_{L^{2} Q^{2} H^{2}}^{(3) \mu n n}=\left(\bar{\ell}_{e} \tau^{I} \gamma^{\alpha} \ell_{\mu}\right)\left(\bar{q}_{n} \tau^{I} \gamma_{\alpha} q_{n}\right)\left(H^{\dagger} H\right) \\
& \mathcal{O}_{L^{2} Q^{2} H^{2}}^{(4) e \mu n n}=\left(\bar{\ell}_{e} \gamma^{\mu} \ell_{\mu}\right)\left(\bar{q}_{n} \tau^{I} \gamma_{\mu} q_{n}\right)\left(H^{\dagger} \tau^{I} H\right) \\
& \mathcal{O}_{L^{2} Q^{2} H^{2}}^{(5) \mu n n}=\epsilon^{I J K}\left(\bar{\ell}_{e} \tau^{I} \gamma^{\mu} \ell_{\mu}\right)\left(\bar{q}_{n} \tau^{J} \gamma_{\mu} q_{n}\right)\left(H^{\dagger} \tau^{K} H\right) \\
& \mathcal{O}_{L^{2} U^{2} H^{2}}^{(1) e \mu n n}=\left(\bar{\ell}_{e} \gamma^{\alpha} \ell_{\mu}\right)\left(\bar{u}_{n} \gamma_{\mu} u_{n}\right)\left(H^{\dagger} H\right) \\
& \mathcal{O}_{L^{2} U^{2} H^{2}}^{(2) \mu n n}=\left(\bar{\ell}_{e} \tau^{I} \gamma^{\alpha} \ell_{\mu}\right)\left(\bar{u}_{k} \gamma_{\alpha} u_{l}\right)\left(H^{\dagger} \tau^{I} H\right) \\
& \mathcal{O}_{L^{2} D^{2} H^{2}}^{(1) e \mu n n}=\left(\bar{\ell}_{e} \gamma^{\alpha} \ell_{\mu}\right)\left(\bar{d}_{k} \gamma_{\alpha} d_{l}\right)\left(H^{\dagger} H\right) \\
& \mathcal{O}_{L^{2} D^{2} H^{2}}^{(2) \mu n n}=\left(\bar{\ell}_{e} \tau^{I} \gamma^{\alpha} \ell_{\mu}\right)\left(\bar{d}_{n} \gamma_{\alpha} d_{n}\right)\left(H^{\dagger} \tau^{I} H\right) \\
& \mathcal{O}_{E^{2} Q^{2} H^{2}}^{(1) e \mu n n}=\left(\bar{e}_{e} \gamma^{\alpha} e_{\mu}\right)\left(\bar{q}_{n} \gamma_{\alpha} q_{n}\right)\left(H^{\dagger} H\right) \\
& \mathcal{O}_{E^{2} Q^{2} H^{2}}^{(2) e \mu n n}=\left(\bar{e}_{e} \gamma^{\alpha} e_{\mu}\right)\left(\bar{q}_{n} \tau^{I} \gamma_{\alpha} q_{n}\right)\left(H^{\dagger} \tau^{I} H\right) \\
& \mathcal{O}_{E^{2} U^{2} H^{2}}^{e \mu n n}=\left(\bar{e}_{e} \gamma^{\alpha} e_{\mu}\right)\left(\bar{u}_{n} \gamma_{\alpha} u_{n}\right)\left(H^{\dagger} H\right) \\
& \mathcal{O}_{E^{2} D^{2} H^{2}}^{e \mu n n}=\left(\bar{e}_{e} \gamma^{\alpha} e_{\mu}\right)\left(\bar{d}_{n} \gamma_{\alpha} d_{n}\right)\left(H^{\dagger} H\right) \\
& \mathcal{O}_{L E D Q H^{2}}^{(1) e \mu n n}=\left(\bar{\ell}_{e} e_{\mu}\right)\left(\bar{d}_{n} q_{n}\right)\left(H^{\dagger} H\right) \\
& \mathcal{O}_{L E D Q H^{2}}^{(2) e \mu n n}=\left(\bar{\ell}_{e} e_{\mu}\right) \tau^{I}\left(\bar{d}_{n} q_{n}\right)\left(H^{\dagger} \tau^{I} H\right) \\
& \mathcal{O}_{L E Q U H^{2}}^{(1) e \mu n n}=\left(\bar{\ell}_{e} e_{\mu}\right) \epsilon\left(\bar{q}_{n} u_{n}\right)\left(H^{\dagger} H\right) \\
& \mathcal{O}_{L E Q U H^{2}}^{(2) e \mu n n}=\left(\bar{\ell}_{e} e_{\mu}\right) \tau^{I} \epsilon\left(\bar{q}_{n} u_{n}\right)\left(H^{\dagger} \tau^{I} H\right) \\
& \mathcal{O}_{L E Q U H^{2}}^{(3) e \mu n n}=\left(\bar{\ell}_{e} \sigma^{\alpha \beta} e_{\mu}\right) \epsilon\left(\bar{q}_{n} \sigma_{\alpha \beta} u_{n}\right)\left(H^{\dagger} H\right) \\
& \mathcal{O}_{L E Q U H^{2}}^{(4) e \mu n n}=\left(\bar{\ell}_{e} \sigma^{\alpha \beta} e_{j}\right) \tau^{I} \epsilon\left(\bar{q}_{n} \sigma_{\alpha \beta} u_{n}\right)\left(H^{\dagger} \tau^{I} H\right)
\end{aligned}
$$

where $n=1,2,3$ runs over the quark generation space.

\section{A.2.2 Two-lepton operators}

Two-lepton operators include the eight dimensional dipoles

$$
\begin{aligned}
\mathcal{O}_{L E W H^{3}}^{(1) e \mu} & =\left(\bar{\ell}_{e} \tau^{I} H \sigma^{\alpha \beta} e_{\mu}\right) W_{\alpha \beta}^{I}\left(H^{\dagger} H\right) \\
\mathcal{O}_{L E W H^{3}}^{(2) e \mu} & =\left(\bar{\ell}_{e} H \sigma^{\alpha \beta} e_{\mu}\right) W_{\alpha \beta}^{I}\left(H^{\dagger} \tau^{I} H\right) \\
\mathcal{O}_{L E B H^{3}}^{e \mu} & =\left(\bar{\ell}_{i} H \sigma^{\alpha \beta} e_{j}\right) B_{\alpha \beta}\left(H^{\dagger} H\right)
\end{aligned}
$$

and the following operators

$$
\begin{aligned}
& \mathcal{O}_{L^{2} H^{4} D}^{(1) e \mu}=i\left(\bar{\ell}_{e} \gamma^{\alpha} \ell_{\mu}\right)\left(H^{\dagger} \stackrel{\leftrightarrow}{D}_{\alpha} H\right)\left(H^{\dagger} H\right) \\
& \mathcal{O}_{L^{2} H^{4} D}^{(2) \mu}=i\left(\bar{\ell}_{e} \tau^{I} \gamma^{\alpha} \ell_{\mu}\right)\left[\left(H^{\dagger} \stackrel{\leftrightarrow}{D_{\alpha}^{I}} H\right)\left(H^{\dagger} H\right)+\left(H^{\dagger} \stackrel{\leftrightarrow}{D}_{\alpha} H\right)\left(H^{\dagger} \tau^{I} H\right)\right] \\
& \mathcal{O}_{E^{2} H^{4} D}^{e \mu}=i\left(\bar{e}_{e} \gamma^{\alpha} e_{\mu}\right)\left(H^{\dagger} \stackrel{\leftrightarrow}{D}_{\alpha} H\right)\left(H^{\dagger} H\right) \quad \mathcal{O}_{L E H^{5}}^{e \mu}=\left(\bar{\ell}_{e} H e_{\mu}\right)\left(H^{\dagger} H\right)^{2},
\end{aligned}
$$


where

$$
\begin{aligned}
& i H^{\dagger} \stackrel{\leftrightarrow}{D_{\mu}} H \equiv i H^{\dagger}\left(D_{\mu} H\right)-i\left(D_{\mu} H^{\dagger}\right) H \\
& i H^{\dagger} \stackrel{\leftrightarrow}{D_{\mu}^{I}} H \equiv i H^{\dagger} \tau^{I}\left(D_{\mu} H\right)-i\left(D_{\mu} H^{\dagger}\right) \tau^{I} H .
\end{aligned}
$$

Following Electroweak Spontaneous Symmetry Breaking, the second set of operators are matched onto four fermion contact interactions at low energy, after integrating out the heavy $Z, h$ bosons at $m_{W}$.

\section{B Tree matching at $m_{W}$ with LFV operators to dimension eight}

This section presents the tree level matching conditions at $m_{W}$ of $\mu \leftrightarrow e$ flavour-changing SMEFT operators, including the dimension eight operators listed in the previous section, but neglecting double-insertions of dimension six operators. The operator basis below $m_{W}$ is given in the notation of $[10,56]$.

\section{B.1 Dipoles and two-photon(gluon)}

Below $m_{W}$, there are the dipole operators of two chiralities, and operators with two photons or two gluons. Above $m_{W}$, there is a dimension six dipole operator for hypercharge, and another one for $\mathrm{SU}(2)$.

Since the photon is the combination $A_{\mu}=\cos \theta_{W} B_{\mu}+\sin \theta_{W} W_{\mu}^{3} \equiv c_{W} B_{\mu}+s_{W} W_{\mu}^{3}$, the low energy dipole coefficient (on the left) is matched onto the dimension six and eight SMEFT dipoles (on the right) as

$$
\begin{aligned}
& C_{D, R}^{e \mu}=c_{W}\left(C_{E B}^{e \mu}+\frac{v^{2}}{y_{\mu} \Lambda_{\mathrm{NP}}^{2}} C_{L E B H^{3}}^{e \mu}\right)-s_{W}\left[C_{E W}^{e \mu}+\frac{v^{2}}{y_{\mu} \Lambda_{\mathrm{NP}}^{2}}\left(C_{L E W H^{3}(1)}^{e \mu}+C_{L E W H^{3}(2)}^{e \mu}\right)\right] \\
& C_{D, L}^{e \mu}=c_{W}\left(C_{E B}^{\mu e *}+\frac{v^{2}}{y_{\mu} \Lambda_{\mathrm{NP}}^{2}} C_{L E B H^{3}}^{\mu e *}\right)-s_{W}\left[C_{E W}^{\mu e *}+\frac{v^{2}}{y_{\mu} \Lambda_{\mathrm{NP}}^{2}}\left(C_{L E W H^{3}(1)}^{\mu e *}+C_{L E W H^{3}(2)}^{\mu e *}\right)\right]
\end{aligned}
$$

where the - sign is due to the $\tau^{3}$ matrix. In addition, since matching "at tree level" mean tree-level in the low-energy theory, loop diagrams in the theory above $m_{W}$ composed of heavy particles can be included. We follow [56] (see [57] for a more recent calculation), and retain the two-loop Barr-Zee diagrams, in which a Higgs leg connect a $W$ or $t$ loop with the neutral Higgs flavour changing vertex of eq. (2.6), and the one loop $Z$-exchange diagram where one $Z$ vertex is flavour changing. The former give the matching condition

$$
\Delta C_{D, L}^{e \mu}\left(m_{W}\right) \simeq-C_{E H}^{\mu e *}\left(m_{W}\right)\left[\frac{e \alpha}{16 \pi^{3} y_{\mu}}\left(Q_{t}^{2} N_{c} Y_{t}^{2}-\frac{7}{2}\right)\right] \simeq C_{E H}^{\mu e *}\left(m_{W}\right)\left[\frac{e \alpha}{8 \pi^{3} y_{\mu}}\right]
$$

while the latter give

$$
\begin{aligned}
\Delta C_{D, L}^{e \mu}\left(m_{W}\right) & \simeq \frac{e}{16 \pi^{2}} g_{L}^{e} C_{H E}^{e \mu}\left(m_{W}\right) \\
\Delta C_{D, R}^{e \mu}\left(m_{W}\right) & \simeq \frac{e}{16 \pi^{2}} g_{R}^{e}\left(C_{H L(1)}^{e \mu}\left(m_{W}\right)+C_{H L(3)}^{e \mu}\left(m_{W}\right)\right),
\end{aligned}
$$


where $g_{L}^{e}, g_{R}^{e}$ are defined in the Feynman rule for $Z$ couplings to leptons $-i \frac{g}{2 c_{W}}\left(g_{L}^{e} P_{L}+\right.$ $\left.g_{R}^{e} P_{R}\right)$ as $g_{R}^{e}=2 s_{W}^{2}$, and $g_{L}^{e}=-1+2 s_{W}^{2}$.

For the two-photon and two-gluon operators the matching conditions are

$$
\begin{aligned}
C_{F F, R}^{e \mu} & =\frac{v}{\Lambda_{\mathrm{NP}}}\left(c_{W}^{2} C_{L E B^{2} H(1)}^{e \mu}-s_{W} c_{W} C_{L E W B H(1)}^{e \mu}+s_{W}^{2} C_{L E W^{2} H(1)}^{e \mu}\right) \\
C_{F F, L}^{e \mu} & =\frac{v}{\Lambda_{\mathrm{NP}}}\left(c_{W}^{2} C_{L E B^{2} H(1)}^{\mu e *}-s_{W} c_{W} C_{L E W B H(1)}^{\mu e *}+s_{W}^{2} C_{L E W^{2} H(1)}^{\mu e *}\right) \\
C_{F \tilde{F}, R}^{e \mu} & =\frac{v}{\Lambda_{\mathrm{NP}}}\left(c_{W}^{2} C_{L E B^{2} H(2)}^{e \mu}-s_{W} c_{W} C_{L E W B H(2)}^{e \mu}+s_{W}^{2} C_{L E W^{2} H(2)}^{e \mu}\right) \\
C_{F \tilde{F}, L}^{e \mu} & =\frac{v}{\Lambda_{\mathrm{NP}}}\left(c_{W}^{2} C_{L E B^{2} H(2)}^{\mu e *}-s_{W} c_{W} C_{L E W B H(2)}^{\mu e *}+s_{W}^{2} C_{L E W^{2} H(2)}^{\mu e *}\right) \\
C_{G G, R}^{e \mu} & =\frac{v}{\Lambda_{\mathrm{NP}}} C_{L E G^{2} H(1)}^{e \mu} \quad C_{G G, L}^{e \mu}=\frac{v}{\Lambda_{\mathrm{NP}}} C_{L E G^{2} H(1)}^{\mu e *} \\
C_{G \tilde{G}, R}^{e \mu} & =\frac{v}{\Lambda_{\mathrm{NP}}} C_{L E G^{2} H(2)}^{e \mu} \quad C_{G \tilde{G}, L}^{e \mu}=\frac{v}{\Lambda_{\mathrm{NP}}} C_{L E G^{2} H(2)}^{\mu e *}
\end{aligned}
$$

\section{B.2 Four-Lepton}

SMEFT operators with four-fermion legs are matched onto four-fermion contact interactions in the low-energy effective theory as Electroweak symmetry is spontaneously broken and the Higgs doublet is replaced by its vacuum expectation value. In addition, given that the interesting LFV operators are $\mu \leftrightarrow e$ flavour changing but otherwise flavour diagonal, two-lepton $\mu \leftrightarrow e$ operators can be connected to a renormalizable vertex exchanging an $h$ or a $Z$, generating an effective four-fermion interaction when the heavy SM bosons are integrated out at the Electroweak scale.

As discussed in the text, a flavour changing vertex with the $h$ Higgs boson appears as the SMEFT operators $\mathcal{O}_{E H}$ and $\mathcal{O}_{L E H^{5}}$ contribute to the leptons mass

$$
\left[m_{e}\right]^{i j}=v\left(\left[Y_{e}\right]^{i j}-C_{E H}^{i j} \frac{v^{2}}{\Lambda_{\mathrm{NP}}^{2}}-C_{L E H^{5}}^{i j} \frac{v^{4}}{\Lambda_{\mathrm{NP}}^{4}}\right),
$$

so that in the charged leptons mass basis the Yukawa coupling is off-diagonal

$$
\begin{aligned}
& \frac{h}{\sqrt{2}} \bar{e}^{i} P_{R} e^{j}\left(\left[Y_{e}\right]^{i j}-3 C_{E H}^{i j} \frac{v^{2}}{\Lambda_{\mathrm{NP}}^{2}}-5 C_{L E H^{5}}^{i j} \frac{v^{4}}{\Lambda_{\mathrm{NP}}^{4}}\right) \\
& =\frac{h}{\sqrt{2}} \bar{e}^{i} P_{R} e^{j}\left(\left[m_{e}\right]^{i j}-2 C_{E H}^{i j} \frac{v^{2}}{\Lambda_{\mathrm{NP}}^{2}}-4 C_{L E H^{5}}^{i j} \frac{v^{4}}{\Lambda_{\mathrm{NP}}^{4}}\right)
\end{aligned}
$$

and the LFV Feynman rule with the neutral Higgs reads

$$
-i \sqrt{2} \bar{e}^{i} P_{R} e^{j}\left(C_{E H}^{i j} \frac{v^{2}}{\Lambda_{\mathrm{NP}}^{2}}+2 C_{L E H^{5}}^{i j} \frac{v^{4}}{\Lambda_{\mathrm{NP}}^{4}}\right)
$$

In SMEFT there are more distinct flavour structures which are matched into the same low energy operators: for example $\mathcal{O}_{L L}^{e \mu f f}, \mathcal{O}_{L L}^{f f e \mu}, \mathcal{O}_{L L}^{f \mu e f}$ and $\mathcal{O}_{L L}^{e f f \mu}$ all match onto the below$m_{W}$ LFV operator $\mathcal{O}_{L L}^{e \mu f f}$. In the following, we suppress these permutations for brevity, and write

$$
C_{\text {low energy }}^{e \mu f f}=C_{\mathrm{SMEFT}}^{e \mu f f}+\text { perm. }
$$


to indicate that these different flavour structures are to be summed on the right side of the matching conditions. These are:

$$
\begin{aligned}
& C_{V, R R}^{e \mu \ell \ell}=C_{E E}^{e \mu \ell \ell}+C_{H E}^{e \mu} g_{R}^{e}+\frac{v^{2}}{\Lambda_{\mathrm{NP}}^{2}}\left(C_{E^{4} H^{2}}^{e \mu \ell \ell}+C_{E^{2} H^{4} D}^{e \mu} g_{R}^{e}\right)+\text { perm. } \\
& C_{V, L R}^{e \mu \ell \ell}=C_{L E}^{e \mu \ell \ell}+\left(C_{H L, 3}^{e \mu}+C_{H L, 1}^{e \mu}\right) g_{R}^{e} \\
& +\frac{v^{2}}{\Lambda_{\mathrm{NP}}^{2}}\left[C_{L^{2} E^{2} H^{2}(1)}^{e \mu l l}+C_{L^{2} E^{2} H^{2}(2)}^{e \mu l l}+\left(C_{L^{2} H^{4} D(1)}^{e \mu}+2 C_{L^{2} H^{4} D(2)}^{e \mu}\right) g_{R}^{e}\right] \\
& C_{V, R L}^{e \mu \ell \ell}=C_{L E}^{\ell \ell e \mu}+C_{H E}^{e \mu} g_{L}^{e}+\frac{v^{2}}{\Lambda_{\mathrm{NP}}^{2}}\left[C_{L^{2} E^{2} H^{2}(1)}^{\ell \ell e \mu}+C_{L^{2} E^{2} H^{2}(2)}^{\ell \ell e \mu}+C_{E^{2} H^{4} D}^{e \mu} g_{L}^{e}\right] \\
& C_{V, L L}^{e \mu l \ell}=C_{L L}^{e \mu l \ell}+\left(C_{H L, 3}^{e \mu}+C_{H L, 1}^{e \mu}\right) g_{L}^{e} \\
& +\frac{v^{2}}{\Lambda_{\mathrm{NP}}^{2}}\left[C_{L^{4} H^{2}(1)}^{e \mu \ell}+C_{L^{4} H^{2}(2)}^{e \mu l \ell}+\left(C_{L^{2} H^{4} D(1)}^{e \mu}+C_{L^{2} H^{4} D(2)}^{e \mu}\right) g_{L}^{e}\right]+\text { perm. } \\
& C_{S, R R}^{e \mu \ell \ell}=-\frac{m_{\ell} C_{E H}^{e \mu} v}{m_{h}^{2}}+\frac{v^{2}}{\Lambda_{\mathrm{NP}}^{2}}\left(C_{L^{2} E^{2} H^{2}(3)}^{e \mu \ell}-2 \frac{m_{\ell} C_{L E H^{5}}^{e \mu} v}{m_{h}^{2}}\right)+\text { perm. } \\
& C_{S, L R}^{e \mu \tau \tau}=-2 C_{L E}^{\tau \mu e \tau}-\frac{m_{\tau} C_{E H}^{\mu e *} v}{m_{h}^{2}}-\frac{v^{2}}{\Lambda_{\mathrm{NP}}^{2}}\left[2\left(C_{L^{2} E^{2} H^{2}(1)}^{\tau \mu e \tau}+C_{L^{2} E^{2} H^{2}(2)}^{\tau \mu e \tau}\right)+2 \frac{m_{\tau} C_{L E H^{5}}^{\mu e *} v}{m_{h}^{2}}\right] \\
& C_{S, R L}^{e \mu \tau \tau}=-2 C_{L E}^{e \tau \tau \mu}-\frac{m_{\tau} C_{E H}^{e \mu} v}{m_{h}^{2}}-\frac{v^{2}}{\Lambda_{\mathrm{NP}}^{2}}\left[2\left(C_{L^{2} E^{2} H^{2}(1)}^{e \tau+\mu} C_{L^{2} E^{2} H^{2}(2)}^{e \tau \tau \mu}\right)+2 \frac{m_{\tau} C_{L E H^{5}}^{e \mu} v}{m_{h}^{2}}\right] \\
& C_{S, L L}^{e \mu \ell \ell}=-\frac{m_{\ell} C_{E H}^{\mu e *} v}{m_{h}^{2}}+\frac{v^{2}}{\Lambda_{\mathrm{NP}}^{2}}\left(C_{L^{2} E^{2} H^{2}(3)}^{\mu e \ell *}-2 \frac{m_{\ell} C_{L E H^{5}}^{\mu e *}}{m_{h}^{2}}\right)+\text { perm. } \\
& C_{T, R R}^{e \mu \tau \tau}=\frac{v^{2}}{\Lambda_{\mathrm{NP}}^{2}} C_{L^{2} E^{2} H^{2}(4)}^{e \mu \tau} \\
& C_{T, L L}^{e \mu \tau \tau}=\frac{v^{2}}{\Lambda_{\mathrm{NP}}^{2}} C_{L^{2} E^{2} H^{2}(4)}^{\mu e \tau \tau *}
\end{aligned}
$$

where $\ell \in\{e, \mu, \tau\}$. We see that lepton tensors are matched at tree level only at dimension eight, and also that dimension eight operators could be significant for LL or RR scalars, where the dimension six contribution is Yukawa-suppressed.

\section{B.3 Two-lepton two-quark}

Given that the low energy constraints are expressed in the quark mass eigenstate basis, in the "bottom up" approach adopted here, the CKM matrix will act on SMEFT operator coefficients in the matching conditions. As we work in the $u_{L}$-basis, a CKM weighted sum will appear in matching $d_{L}$ operators.(The CKM matrix is here written as $V$, rather than the previously used $V_{C K M}$.) 
Tree-level matching SMEFT dimension six and eight coefficients (on the right) onto low energy coeffic ients (on the left) results in:

$$
\begin{aligned}
& C_{L L}^{e \mu u_{n} u_{n}}=C_{L Q(1)}^{e \mu n n}-C_{L Q(3)}^{e \mu n n}+g_{L}^{u}\left(C_{H L(1)}^{e \mu}+C_{H L(3)}^{e \mu}\right) \\
& +\frac{v^{2}}{\Lambda_{\mathrm{NP}}^{2}}\left[C_{L^{2} Q^{2} H^{2}(1)}^{e \mu n n}+C_{L^{2} Q^{2} H^{2}(2)}^{e \mu n n}-C_{L^{2} Q^{2} H^{2}(3)}^{e \mu n n}-C_{L^{2} Q^{2} H^{2}(4)}^{e \mu n n}\right. \\
& \left.+\left(C_{L^{2} H^{4} D(1)}^{e \mu}+2 C_{L^{2} H^{4} D(2)}^{e \mu}\right) g_{L}^{u}\right] \\
& C_{L L}^{e \mu d_{n} d_{n}}=\sum_{j k} V_{j n} V_{k n}^{*}\left(C_{L Q(1)}^{e \mu j k}+C_{L Q(3)}^{e \mu j k}\right)+g_{L}^{d}\left(C_{H L(1)}^{e \mu}+C_{H L(3)}^{e \mu}\right) \\
& +\frac{v^{2}}{\Lambda_{\mathrm{NP}}^{2}}\left[\sum_{j k} V_{j n} V_{k n}^{*}\left(C_{L^{2} Q^{2} H^{2}(1)}^{e \mu j k}+C_{L^{2} Q^{2} H^{2}(2)}^{e \mu j k}+C_{L^{2} Q^{2} H^{2}(3)}^{e \mu j k}+C_{L^{2} Q^{2} H^{2}(4)}^{e \mu j k}\right)\right. \\
& \left.+\left(C_{L^{2} H^{4} D(1)}^{e \mu}+2 C_{L^{2} H^{4} D(2)}^{e \mu}\right) g_{L}^{d}\right] \\
& C_{R R}^{e \mu u_{n} u_{n}}=C_{E U}^{e \mu n n}+g_{R}^{u} C_{H E}^{e \mu}+\frac{v^{2}}{\Lambda_{\mathrm{NP}}^{2}}\left(C_{E^{2} U^{2} H^{2}}^{e \mu n n}+C_{E^{2} H^{4} D}^{e \mu} g_{R}^{u}\right) \\
& C_{R R}^{e \mu d_{n} d_{n}}=C_{E D}^{e \mu n n}+g_{R}^{d} C_{H E}^{e \mu}+\frac{v^{2}}{\Lambda_{\mathrm{NP}}^{2}}\left(C_{E^{2} D^{2} H^{2}}^{e \mu n n}+C_{E^{2} H^{4} D}^{e \mu} g_{R}^{d}\right) \\
& C_{L R}^{e \mu u_{n} u_{n}}=C_{L U}^{e \mu n n}+g_{R}^{u}\left(C_{H L(1)}^{e \mu}+C_{H L(3)}^{e \mu}\right) \\
& +\frac{v^{2}}{\Lambda_{\mathrm{NP}}^{2}}\left[C_{L^{2} U^{2} H^{2}(1)}^{e \mu n n}+C_{L^{2} U^{2} H^{2}(2)}^{e \mu n n}+\left(C_{L^{2} H^{4} D(1)}^{e \mu}+2 C_{L^{2} H^{4} D(2)}^{e \mu}\right) g_{R}^{u}\right] \\
& C_{L R}^{e \mu d_{n} d_{n}}=C_{L D}^{e \mu n n}+g_{R}^{d}\left(C_{H L(1)}^{e \mu}+C_{H L(3)}^{e \mu}\right) \\
& +\frac{v^{2}}{\Lambda_{\mathrm{NP}}^{2}}\left[C_{L^{2} D^{2} H^{2}(1)}^{e \mu n n}+C_{L^{2} D^{2} H^{2}(2)}^{e \mu n n}+\left(C_{L^{2} H^{4} D(1)}^{e \mu}+2 C_{L^{2} H^{4} D(2)}^{e \mu}\right) g_{R}^{d}\right] \\
& C_{R L}^{e \mu u_{n} u_{n}}=C_{E Q}^{e \mu n n}+g_{L}^{u} C_{H E}^{e \mu}+\frac{v^{2}}{\Lambda_{\mathrm{NP}}^{2}}\left[C_{E^{2} Q^{2}(1)}^{e \mu n n}-C_{E^{2} Q^{2}(2)}^{e \mu n n}+C_{E^{2} H^{4} D}^{e \mu} g_{L}^{u}\right] \\
& C_{R L}^{e \mu d_{n} d_{n}}=\sum_{j k} V_{j n} V_{k n}^{*} C_{E Q}^{e \mu j k}+g_{L}^{d} C_{H E}^{e \mu} \\
& +\frac{v^{2}}{\Lambda_{\mathrm{NP}}^{2}}\left[\sum_{j k} V_{j n} V_{k n}^{*}\left(C_{E^{2} Q^{2}(1)}^{e j k}+C_{E^{2} Q^{2}(2)}^{e \mu j k}\right)+C_{E^{2} H^{4} D}^{e \mu} g_{L}^{d}\right] \\
& C_{S, L L}^{e \mu u_{n} u_{n}}=-C_{L E Q U}^{* \mu e n n}-\frac{m_{u_{n} v}}{m_{h}^{2}} C_{E H}^{\mu e *}-\frac{v^{2}}{\Lambda_{\mathrm{NP}}^{2}}\left(C_{L E Q U H^{2}(1)}^{\mu e n n *}+C_{L E Q U H^{2}(2)}^{\mu e n n *}+2 \frac{m_{u_{n}} v}{m_{h}^{2}} C_{L E H^{5}}^{\mu e *}\right) \\
& C_{S, L L}^{e \mu d_{n} d_{n}}=-\frac{m_{d_{n}} v}{m_{h}^{2}} C_{E H}^{\mu e *}+\frac{v^{2}}{\Lambda_{\mathrm{NP}}^{2}}\left(\sum_{j} V_{j n}^{*} C_{L E Q D H^{2}(3)}^{\mu e j n *}-2 \frac{m_{d_{n}} v}{m_{h}^{2}} C_{L E H^{5}}^{\mu e *}\right) \\
& C_{S, R R}^{e \mu u_{n} u_{n}}=-C_{L E Q U}^{e \mu n n}-\frac{m_{u_{n}} v}{m_{h}^{2}} C_{E H}^{e \mu}-\frac{v^{2}}{\Lambda_{\mathrm{NP}}^{2}}\left(C_{L E Q U H^{2}(1)}^{e \mu n n}+C_{L E Q U H^{2}(2)}^{e \mu n n}+2 \frac{m_{u_{n}} v}{m_{h}^{2}} C_{L E H^{5}}^{e \mu}\right)
\end{aligned}
$$




$$
\begin{aligned}
C_{S, R R}^{e \mu d_{n} d_{n}}= & -\frac{m_{d_{n}} v}{m_{h}^{2}} C_{E H}^{e \mu}+\frac{v^{2}}{\Lambda_{\mathrm{NP}}^{2}}\left(\sum_{j} V_{j n} C_{L E Q D H^{2}(3)}^{e \mu j n}-2 \frac{m_{d_{n}} v}{m_{h}^{2}} C_{L E H^{5}}^{e \mu}\right) \\
C_{S, L R}^{e \mu u_{n} u_{n}}= & -\frac{m_{u_{n}} v}{m_{h}^{2}} C_{E H}^{\mu e *}-\frac{v^{2}}{\Lambda_{\mathrm{NP}}^{2}}\left(2 \frac{m_{u_{n} v} v}{m_{h}^{2}} C_{L E H^{5}}^{\mu e *}-C_{L E Q U H^{2}(5)}^{\mu e n n *}\right) \\
C_{S, L R}^{e \mu d_{n} d_{n}}= & \sum_{j} V_{j n} C_{L E D Q}^{* \mu e n j}-\frac{m_{d_{n}} v}{m_{h}^{2}} C_{E H}^{\mu e *} \\
& +\frac{v^{2}}{\Lambda_{\mathrm{NP}}^{2}}\left[\sum_{j} V_{j n}\left(C_{L E Q D H^{2}(1)}^{\mu e n j *}+C_{L E Q D H^{2}(2)}^{\mu e n j *}\right)-2 \frac{m_{d_{n}} v}{m_{h}^{2}} C_{L E H^{5}}^{\mu e *}\right] \\
C_{S, R L}^{e \mu u_{n} u_{n}}= & -\frac{m_{u_{n}} v}{m_{h}^{2}} C_{E H}^{e \mu}-\frac{v^{2}}{\Lambda_{\mathrm{NP}}^{2}}\left(2 \frac{m_{u_{n}} v}{m_{h}^{2}} C_{L E H^{5}}^{e \mu}-C_{L E Q U H^{2}(5)}^{e \mu n n}\right) \\
C_{S, R L}^{e \mu d_{n} d_{n}}= & \sum_{j} V_{j n}^{*} C_{L E D Q}^{e \mu n j}-\frac{m_{d_{n}} v}{m_{h}^{2}} C_{E H}^{e \mu} \\
& +\frac{v^{2}}{\Lambda_{\mathrm{NP}}^{2}}\left[\sum_{j} V_{j n}^{*}\left(C_{L E Q D H^{2}(1)}^{e \mu n j}+C_{L E Q D H^{2}(2)}^{e \mu n j}\right)-2 \frac{m_{d_{n}} v}{m_{h}^{2}} C_{L E H^{5}}^{e \mu}\right] \\
C_{T, L L}^{e \mu u_{n} u_{n}}= & -C_{T, L E Q U}^{* \mu n n}-\frac{v^{2}}{\Lambda_{\mathrm{NP}}^{2}}\left(C_{L E Q U H^{2}(3)}^{\mu e n n *}+C_{L E Q U H^{2}(4)}^{\mu e n n *}\right) \\
C_{T, R R}^{e \mu u_{n} u_{n}}= & -C_{T, L E Q U}^{e \mu n n}-\frac{v^{2}}{\Lambda_{\mathrm{NP}}^{2}}\left(C_{L E Q U H^{2}(3)}^{e \mu n n}+C_{L E Q U H^{2}(4)}^{e \mu n n}\right) \\
C_{T, R R}^{e \mu d_{n} d_{n}}= & \frac{v^{2}}{\Lambda_{\mathrm{NP}}^{2}} \sum_{j} V_{j n} C_{L E Q D H^{2}(5)}^{e \mu j n} \\
C_{T, L L}^{e \mu d_{n} d_{n}}= & \frac{v^{2}}{\Lambda_{\mathrm{NP}}^{2}} \sum_{j} V_{j n}^{*} C_{L E Q D H^{2}(5)}^{\mu e j n *}
\end{aligned}
$$

where $V$ is the CKM matrix, $u_{n} \in\{u, c\}, d_{n} \in\{d, s, b\}$, and

$$
g_{L}^{u}=1-\frac{4}{3} s_{W}^{2}, \quad g_{R}^{u}=-\frac{4}{3} s_{W}^{2}, \quad g_{L}^{d}=-1+\frac{2}{3} s_{W}^{2}, \quad g_{R}^{d}=\frac{2}{3} s_{W}^{2} .
$$

As anticipated, the low energy LFV tensors involving $d$-type quarks are matched at tree level onto the SMEFT eight dimensional tensors. Dimension eight operators could also be relevant for LL, RR scalars with $d$ quarks and RL, LR scalars with $u$ quarks, as the dimension six contributions are suppressed by Yukawa couplings.

Open Access. This article is distributed under the terms of the Creative Commons Attribution License (CC-BY 4.0), which permits any use, distribution and reproduction in any medium, provided the original author(s) and source are credited.

\section{References}

[1] W. Buchmüller and D. Wyler, Effective Lagrangian Analysis of New Interactions and Flavor Conservation, Nucl. Phys. B 268 (1986) 621 [INSPIRE].

[2] B. Grzadkowski, M. Iskrzynski, M. Misiak and J. Rosiek, Dimension-Six Terms in the Standard Model Lagrangian, JHEP 10 (2010) 085 [arXiv:1008.4884] [INSPIRE]. 
[3] Y. Kuno and Y. Okada, Muon decay and physics beyond the standard model, Rev. Mod. Phys. 73 (2001) 151 [hep-ph/9909265] [INSPIRE].

[4] J.D. Bjorken and S. Weinberg, A Mechanism for Nonconservation of Muon Number, Phys. Rev. Lett. 38 (1977) 622 [INSPIRE].

[5] S.M. Barr and A. Zee, Electric Dipole Moment of the Electron and of the Neutron, Phys. Rev. Lett. 65 (1990) 21 [Erratum ibid. 65 (1990) 2920] [INSPIRE].

[6] L. Wolfenstein, Parametrization of the Kobayashi-Maskawa Matrix, Phys. Rev. Lett. 51 (1983) 1945.

[7] A.J. Buras, Weak Hamiltonian, CP-violation and rare decays, in Les Houches Summer School in Theoretical Physics, Session 68: Probing the Standard Model of Particle Interactions 6 (1998) [hep-ph/9806471] [INSPIRE].

[8] L. Silvestrini, Effective Theories for Quark Flavour Physics, Les Houches Lect. Notes 108 (2020) [arXiv: 1905. 00798] [INSPIRE].

[9] T. Huber, E. Lunghi, M. Misiak and D. Wyler, Electromagnetic logarithms in $\bar{B} \rightarrow X_{s} l^{+} l^{-}$, Nucl. Phys. B $\mathbf{7 4 0}$ (2006) 105 [hep-ph/0512066] [INSPIRE].

[10] S. Davidson, Completeness and complementarity for $\mu \rightarrow e \gamma \mu \rightarrow e \bar{e} e$ and $\mu A \rightarrow e A$, JHEP 02 (2021) 172 [arXiv:2010.00317] [INSPIRE].

[11] A. Crivellin, S. Davidson, G.M. Pruna and A. Signer, Renormalisation-group improved analysis of $\mu \rightarrow e$ processes in a systematic effective-field-theory approach, JHEP 05 (2017) 117 [arXiv: 1702.03020] [INSPIRE].

[12] C.D. Froggatt and H.B. Nielsen, Hierarchy of Quark Masses, Cabibbo Angles and CP-violation, Nucl. Phys. B 147 (1979) 277 [INSPIRE].

[13] G. D'Ambrosio, G.F. Giudice, G. Isidori and A. Strumia, Minimal flavor violation: An Effective field theory approach, Nucl. Phys. B 645 (2002) 155 [hep-ph/0207036] [InSPIRE].

[14] M. Bordone, O. Catà and T. Feldmann, Effective Theory Approach to New Physics with Flavour: General Framework and a Leptoquark Example, JHEP 01 (2020) 067 [arXiv: 1910.02641] [INSPIRE].

[15] R. Alonso, E.E. Jenkins, A.V. Manohar and M. Trott, Renormalization Group Evolution of the Standard Model Dimension Six Operators III: Gauge Coupling Dependence and Phenomenology, JHEP 04 (2014) 159 [arXiv:1312.2014] [INSPIRE].

[16] E.E. Jenkins, A.V. Manohar and M. Trott, Renormalization Group Evolution of the Standard Model Dimension Six Operators II: Yukawa Dependence, JHEP 01 (2014) 035 [arXiv: 1310.4838] [INSPIRE].

[17] C.W. Murphy, Dimension-8 operators in the Standard Model Eective Field Theory, JHEP 10 (2020) 174 [arXiv: 2005.00059] [INSPIRE].

[18] S. Herrlich and U. Nierste, Evanescent operators, scheme dependences and double insertions, Nucl. Phys. B 455 (1995) 39 [hep-ph/9412375] [INSPIRE].

[19] G.F. Giudice and O. Lebedev, Higgs-dependent Yukawa couplings, Phys. Lett. B 665 (2008) 79 [arXiv: 0804.1753] [INSPIRE].

[20] CMS collaboration, Observation of the Higgs boson decay to a pair of $\tau$ leptons with the CMS detector, Phys. Lett. B 779 (2018) 283 [arXiv:1708.00373] [INSPIRE].

[21] ATLAS collaboration, Cross-section measurements of the Higgs boson decaying into a pair of $\tau$-leptons in proton-proton collisions at $\sqrt{s}=13 \mathrm{TeV}$ with the ATLAS detector, Phys. Rev. D 99 (2019) 072001 [arXiv: 1811.08856] [INSPIRE]. 
[22] ATLAS collaboration, A search for the dimuon decay of the Standard Model Higgs boson with the ATLAS detector, Phys. Lett. B 812 (2021) 135980 [arXiv:2007.07830] [INSPIRE].

[23] CMS collaboration, Evidence for Higgs boson decay to a pair of muons, JHEP 01 (2021) 148 [arXiv: 2009.04363] [INSPIRE].

[24] CMS collaboration, Search for lepton flavour violating decays of the Higgs boson to $\mu \tau$ and eT in proton-proton collisions at $\sqrt{s}=13 \mathrm{TeV}$, JHEP 06 (2018) 001 [arXiv:1712.07173] [INSPIRE].

[25] ATLAS collaboration, Search for lepton-flavour-violating decays of the Higgs and $Z$ bosons with the ATLAS detector, Eur. Phys. J. C 77 (2017) 70 [arXiv:1604.07730] [InSPIRE].

[26] MEG collaboration, Search for the lepton flavour violating decay $\mu^{+} \rightarrow \mathrm{e}^{+} \gamma$ with the full dataset of the MEG experiment, Eur. Phys. J. C 76 (2016) 434 [arXiv:1605.05081] [INSPIRE].

[27] Particle Data Group collaboration, Review of Particle Physics, Chin. Phys. C 38 (2014) 090001 [INSPIRE] and 2015 update.

[28] MEG II collaboration, The design of the MEG II experiment, Eur. Phys. J. C 78 (2018) 380 [arXiv: 1801.04688] [INSPIRE].

[29] SINDRUM collaboration, Search for the Decay $\mu \rightarrow 3 e$, Nucl. Phys. B 299 (1988) 1 [INSPIRE].

[30] A. Blondel et al., Research Proposal for an Experiment to Search for the Decay $\mu \rightarrow$ eee, arXiv: 1301.6113 [INSPIRE].

[31] SINDRUM II collaboration, A Search for muon to electron conversion in muonic gold, Eur. Phys. J. C 47 (2006) 337 [InSPIRE].

[32] SINDRUM II collaboration, Test of lepton flavor conservation in $\mu \rightarrow e$ conversion on titanium, Phys. Lett. B 317 (1993) 631 [INSPIRE].

[33] SINDRUM II collaboration, Improved limit on the branching ratio of $\mu \rightarrow e$ conversion on lead, Phys. Rev. Lett. 76 (1996) 200 [INSPIRE].

[34] Mu2E collaboration, Proposal to search for $\mu^{-} N \rightarrow e^{-} N$ with a single event sensitivity below $10^{-16}$, FERMILAB-PROPOSAL-0973 [inSPIRE].

[35] COMET collaboration, Conceptual design report for experimental search for lepton flavor violating $m u^{-}-e^{-}$conversion at sensitivity of $10^{-16}$ with a slow-extracted bunched proton beam (COMET), KEK-2009-10.

[36] COMET collaboration, Overview of the COMET Phase-I experiment, PoS FPCP2015 (2015) 059 [INSPIRE].

[37] BABAR collaboration, Searches for Lepton Flavor Violation in the Decays $\tau^{ \pm} \rightarrow e^{ \pm} \gamma$ and $\tau^{ \pm} \rightarrow \mu^{ \pm} \gamma$, Phys. Rev. Lett. 104 (2010) 021802 [arXiv:0908.2381] [INSPIRE].

[38] K. Hayasaka et al., Search for Lepton Flavor Violating Tau Decays into Three Leptons with 719 Million Produced Tau+Tau-Pairs, Phys. Lett. B 687 (2010) 139 [arXiv:1001.3221] [INSPIRE].

[39] Belle-II collaboration, The Belle II Physics Book, PTEP 2019 (2019) 123C01 [Erratum ibid. 2020 (2020) 029201] [arXiv: 1808.10567] [INSPIRE].

[40] BeLLE collaboration, Search for lepton flavor violating tau- decays into l- eta, l- eta-prime and l- piO, Phys. Lett. B 648 (2007) 341 [hep-ex/0703009] [INSPIRE]. 
[41] Belle collaboration, Search for Lepton-Flavor-Violating tau Decays into a Lepton and a Vector Meson, Phys. Lett. B 699 (2011) 251 [arXiv:1101.0755] [InSPIRE].

[42] S. Davidson and A. Saporta, Constraints on $2 \ell 2 q$ operators from $\mu-e$ flavour-changing meson decays, Phys. Rev. D 99 (2019) 015032 [arXiv: 1807.10288] [INSPIRE].

[43] PRISM collaboration, An Experimental Search for a $\mu N \rightarrow e N$ Conversion at Sensitivity of the Order of $10^{-18}$ with a Highly Intense Muon Source: PRISM, unpublished, J-PARC LOI (2006).

[44] M. Carpentier and S. Davidson, Constraints on two-lepton, two quark operators, Eur. Phys. J. $C 70$ (2010) 1071 [arXiv: 1008.0280] [InSPIRE].

[45] H.-L. Li, Z. Ren, J. Shu, M.-L. Xiao, J.-H. Yu and Y.-H. Zheng, Complete set of dimension-eight operators in the standard model effective field theory, Phys. Rev. D 104 (2021) 015026 [arXiv: 2005.00008] [INSPIRE].

[46] Y. Farzan and M. Tortola, Neutrino oscillations and Non-Standard Interactions, Front. in Phys. 6 (2018) 10 [arXiv:1710.09360] [inSPIRE].

[47] S. Davidson, Y. Kuno, Y. Uesaka and M. Yamanaka, Probing $\mu$ er $\gamma$ contact interactions with $\mu \rightarrow e$ conversion, Phys. Rev. D 102 (2020) 115043 [arXiv:2007.09612] [INSPIRE].

[48] S. Davidson and M. Gorbahn, Charged lepton flavor change and nonstandard neutrino interactions, Phys. Rev. D 101 (2020) 015010 [arXiv:1909.07406] [INSPIRE].

[49] M. Ciuchini, E. Franco, L. Reina and L. Silvestrini, Leading order QCD corrections to $b \rightarrow s \gamma$ and $b \rightarrow s g$ decays in three regularization schemes, Nucl. Phys. B 421 (1994) 41 [hep-ph/9311357] [INSPIRE].

[50] A. Czarnecki, W.J. Marciano and A. Vainshtein, Refinements in electroweak contributions to the muon anomalous magnetic moment, Phys. Rev. D 67 (2003) 073006 [Erratum ibid. 73 (2006) 119901] [hep-ph/0212229] [INSPIRE].

[51] L.V. Silva, S. Jäger and K. Leslie, Using dipole processes to constrain the flavour of four-fermion effective interactions, PoS ICHEP2020 (2021) 434 [arXiv:2012.05630] [INSPIRE].

[52] G. Buchalla and O. Catà, Effective Theory of a Dynamically Broken Electroweak Standard Model at NLO, JHEP 07 (2012) 101 [arXiv:1203.6510] [INSPIRE].

[53] G. Buchalla, O. Catà and C. Krause, Complete Electroweak Chiral Lagrangian with a Light Higgs at NLO, Nucl. Phys. B 880 (2014) 552 [Erratum ibid. 913 (2016) 475] [arXiv: 1307.5017] [INSPIRE].

[54] A. Pich, I. Rosell, J. Santos and J.J. Sanz-Cillero, Low-energy signals of strongly-coupled electroweak symmetry-breaking scenarios, Phys. Rev. D 93 (2016) 055041 [arXiv: 1510.03114] [INSPIRE].

[55] A. Pich, I. Rosell, J. Santos and J.J. Sanz-Cillero, Fingerprints of heavy scales in electroweak effective Lagrangians, JHEP 04 (2017) 012 [arXiv: 1609.06659] [INSPIRE].

[56] S. Davidson, $\mu \rightarrow e \gamma$ and matching at $m_{W}$, Eur. Phys. J. C 76 (2016) 370 [arXiv: 1601.07166] [INSPIRE].

[57] W. Dekens and P. Stoffer, Low-energy effective field theory below the electroweak scale: matching at one loop, JHEP 10 (2019) 197 [arXiv:1908.05295] [INSPIRE]. 